\title{
Synthesis and Pharmacological Evolution of Tetrahydroisoquinolines as Anti Breast Cancer Agents
}

\author{
Madhavi Gangapuram, Suresh Eyunni and Kinfe K Redda* \\ College of Pharmacy and Pharmaceutical Sciences, Florida A \& M University, Tallahassee, FL 32307, USA
}

\begin{abstract}
Breast cancer is leading cause of mortality among women, resulting in more than half a million deaths worldwide each year. Unfortunately, the recovery rate of advanced breast cancer by current available drug treatment is till unacceptably low. Chemotherapy is the main stay of cancer treatment and most of the drugs cause general toxicity to any non-proliferating cells, which can severely limit the therapeutic values of these drugs. Tetrahydroisoqinoline derivatives (THIQs) were identified as subtype selective estrogen receptor antagonists/agonists hence, potential therapeutic agents for breast cancer. Substituted THIQs were synthesized and well characterized. Antiproliferative activity against human ER (+) MCF-7 (Breast), ER(-) MDA-MB-231 (breast) and Ishikawa (endometrial) cancer cell lines were studied after 72 hours drug exposure employing CellTiter-Glo assay at concentrations ranging from 0.01$100,000 \mathrm{nM}$. The activities of these compounds were compared with Tamoxifen (TAM). In-vitro results indicated that most of the compounds showed better activity than TAM. The most active compounds obtained in this study were $\mathbf{6 a}, \mathbf{6 b}, \mathbf{6 d}$ and $\mathbf{6 j}\left(\mathrm{IC}_{50}=0.63,0.23 ; 0.93,0.21 ; 043,0.01 ; 0.7,0.02 \mu \mathrm{g} / \mathrm{ml}\right)$ against MCF-7 and Ishikawa cell lines, in comparison to Tamoxifen activity $\left(\mathrm{IC}_{50}=5.14,4.55 \mu \mathrm{g} / \mathrm{ml}\right)$. The newly synthesized molecules were docked in the active sites of the ER- $\alpha$ (PDB: 3ERT) and ER- $\beta$ (PDB: 3ERT) crystal structures and probable binding modes of this class of molecules were determined.
\end{abstract}

Keywords: Substituted Tetrahydroisoquinolines (THIQs); Antiproliferative activity; Breast cancer

\section{Introduction}

Breast cancer is the second leading cause of cancer-related deaths in women and is the most common cancer among women, excluding non-melanoma skin cancers. An estimated 232,340 new cases of invasive breast cancer are expected among American women [1]. The nuclear receptors, Estrogen receptor (ER) and progesterone receptor (PR) and their associated steroid hormones are play an important role in the development, differentiation and function of normal breast and endometrial cells. There is strong evidence that estrogen plays an essential role in the growth of breast tumors and the status of these hormones is employed as diagnostic indicators for endocrine responsiveness and tumor recurrence [2]. The estrogen receptors (ERs) remain one of the attractive targets in the treatment of breast cancer till date. Till date, the two subtypes of human ERs identified, ER- $\alpha$ and ER- $\beta$ have different tissue distribution pattern and transcriptional activity. One way of blocking the estrogen action on tumor cells is preventing the binding of estrogen $\left(\mathrm{E}_{2}\right)$ to estrogen receptor (ER) by designing novel inhibitors of ER capable of blocking $\mathrm{E}_{2}$ without showing any estrogenic activity on their own [3-6]. $\mathrm{E}_{2}$ and its derivatives are used by millions of women in Harmone Replacement Therapy (HRT) for the treatment of peri- and post-menopausal related symptoms [7-9]. Steroidal skeleton of $\mathrm{E}_{2}$ was also used as the template for the attachment of various substituents for therapeutic applications in the treatment of harmone-dependent breast cancer [10-11]. In this regard, $\mathrm{E}_{2}$ derivatives like ICI-182,780 Fulvestrant (3) and ICI-164,384 (4) proved to be effective anti breast cancer agents, whose activity is comparable to tamoxifen (TAM) [12,13]. However, steroid based anti-breast cancer agents have their own limitations and hence non-steroidal alternatives were sought. The search for non-steroidal, anti-estrogenic molecules commonly called as Selective estrogen receptor modulators (SERMs) as potential alternatives in the treatment of harmone-dependent $\mathrm{ER}(+)$ breast cancer is actively undertaken in recent years. In this regard, Tamoxifen (TAM) has been the leading drug to treat breast cancer for more than two decades and has proven to be an effective treatment for ER (+) breast cancer, particularly in the post-menopausal women [1417]. However, it is not without adverse side effects. Tamoxifen behaves as ER antagonist in the breast tissue and as ER agonist in bone, and has prophylactic use in breast cancer [18]. Its agonistic effect on the uterus is said to be associated with increased risk of developing endometrial cancer [19]. Thus, alternative chemical entities, preferably non-steroid estrogen receptor modulators are sought with no agonistic effects on uterus.

Tetrahydroisoqinoline derivatives were identified as subtype selective estrogen receptor antagonists/agonists hence, potential therapeutic agents for breast cancer [20,21]. Structure activity relationship studies (SAR) of ER- $\alpha$ selective tetrahydroisoquinolines were reported by Renaud et al. [22]. Tetrahydroisoquinolines incorporating conformationally restricted side chains as the replacement of the amonoethoxy residue of $E_{2}$, typical of SERMs were reported exhibiting binding affinity to ER- $\alpha$ and antagonistic properties [23]. More recently, new steroidomimetic tetrahydroquinolines were reported which act as microtubule disruptors [24].

However, in the development of anti-estrogenic drugs, it is critical that the compounds under study do not cause estrogenic stimulation of the uterus, which could lead to both increase in uterine bleeding

*Corresponding author: Kinfe K. Redda, Ph.D. Professor \& V.P. for Research College of Pharmacy \& Pharmaceutical Sciences, Florida A \& M University, 410 Foote-Hilyer Administration Center, Division of Research, Tallahassee, FL 32307 USA, Tel: 850.412.5102; E-mail: kinfe.redda@famu.edu

Received March 11, 2014; Accepted April 18, 2014; Published April 25, 2014

Citation: Gangapuram M, Eyunni S, Redda KK (2014) Synthesis and Pharmacological Evolution of Tetrahydroisoquinolines as Anti Breast Cancer Agents. J Cancer Sci Ther 6: 161-169. doi:10.4172/1948-5956.1000266

Copyright: @ 2014 Gangapuram M, et al. This is an open-access article distributed under the terms of the Creative Commons Attribution License, which permits unrestricted use, distribution, and reproduction in any medium, provided the original author and source are credited. 
and an increased risk of developing uterine cancer. Ishikawa cell line, a well-differentiated human endometrial adenocarcinoma cell line expresses functional estrogen receptor alpha (ER $)$ and estrogen receptor beta $\left(\mathrm{ER}_{\beta}\right)$ isoforms. Therefore testing the new potential antiproliferative moieties on Ishikawa cell line in the initial stages of the design is a good idea to understand the risk associated in developing endometrial cancer and its treatment. Also the compounds which act as antiproliferative agents against endometrial cancer can be used in combination with Tamoxifen (TAM) in the treatment of breast cancer, particularly in post-menopausal women.

Here in we report the synthesis and in-vitro antiproliferative activity of new tetrahydroisoquinolines (THIQs) against MCF-7, MDA-MB-231 human breast cancer cell lines and Ishikawa human endometrial adenocarcinoma cell lines. These cell lines are widely accepted in vitro models for assessing potent anti-proliferative and anti-estrogenic compounds including SERMs. Tamoxifen (TAM) was used as a standard for comparison of activities in all these studies. An in-silico docking analysis of these compounds in the active sites of the ER- $\alpha$ and ER- $\beta$ crystal structures, ER- $\alpha-4-O H T$ complex (3ERT) and ER $\beta$-RAL complex $(1 \mathrm{QKN})$ and probable binding modes of the molecules in their active sites were determined.

\section{Materials and Methods}

\section{Experimental section}

General: Melting points were determined on a Mel-Temp 3.0 melting point apparatus and are uncorrected. The structures of the products described were confirmed by IR, ${ }^{1} \mathrm{H}$ NMR and elemental analysis data. ${ }^{1} \mathrm{H}$ NMR spectra were recorded on Varian Gemini HX 300 $\mathrm{MHz}$ spectrometer. All chemical shifts expressed in parts per million $(\delta, \mathrm{ppm})$ are reported relative to tetramethylsilane (TMS) as internal standard for solution in $\mathrm{CDCl}_{3}$ as a solvent unless otherwise specified. The IR spectra were run with $\mathrm{KBr}$ pellets on Perkin-Elmer FTIR 1430 spectrometer and are reported in $\mathrm{cm}^{-1}$. Elemental analyses were carried out by Atlantic Microlab, Inc., Norcross, GA, and are within $\pm 0.4 \%$ of theoretical values unless otherwise noted. Flash chromatography was performed on Combi-Flash (Teledyne Isco) using RediSep columns. All Chemicals and solvents were purchased from Sigma-Aldrich and were used without further purification.

General procedure for the synthesis of 2-Aminoisoquinolinium Iodide (3): A solution of hydroxylamine- $O$-sulfonic acid 2 (2 g, 1 equiv), water $(10 \mathrm{~mL})$ and Isoquinoline $1(6.3 \mathrm{~mL}, 3$ equiv) was heated at $90^{\circ} \mathrm{C}$ for $2-3 \mathrm{~h}$. Potassium carbonate $(2.44 \mathrm{~g}, 1$ equiv) was added, and the water was evaporated. Ethanol $(20-30 \mathrm{~mL})$ was added to the solid residue, and insoluble potassium sulfate was filtered out. Hydroiodic acid $(57-67 \%, 1.34 \mathrm{~mL}, 1$ equiv) was added to the filtrated, and the resulting solution was placed in a freezer. The precipitate was filtered out, washed with ethanol, and dried in vacuo. Isolated products were used as such in further reactions. Yield of the product is $1.78 \mathrm{~g}$.

${ }^{1} \mathrm{HNMR}\left(\mathrm{CDCl}_{3}\right) \delta(\mathrm{ppm}): 8.01(\mathrm{td}, J=7.2 \mathrm{~Hz}, 1 \mathrm{H}), 8.12(\mathrm{td}, J=7.2$, $1.2 \mathrm{~Hz}, 1 \mathrm{H}), 8.30(\mathrm{~d}, J=8.1 \mathrm{~Hz}, 1 \mathrm{H}), 8.43\left(\mathrm{~d}, J=8.1 \mathrm{~Hz},-\mathrm{NH}_{2}, \mathrm{D}_{2} \mathrm{O}\right.$ exchange), 8.50-8.57 (m, $4 \mathrm{H}), 9.69(\mathrm{~s}, 1 \mathrm{H})$.

General procedure for acylation (5a-x): To an ice-cold solution of 2-Aminoisoquinolinium iodide $(8 \mathrm{mmol})$ in $35 \mathrm{ml}$ of anhydrous tetrahydrofuran, was added to a substituted acid chloride or substituted benzenesulfonyl chlorides $(12 \mathrm{mmol})$ with stirring. The reaction was allowed to proceed for $12 \mathrm{~h}$ at $70^{\circ} \mathrm{C}$. After cooling to room temperature the reaction was quenched by adding $25 \mathrm{ml}$ of saturated aqueous sodium bicarbonate solution. The mixture was shaken repeatedly in separator funnel and allowed to stand for few minutes. Extraction with dichloromethane $(2 \times 100 \mathrm{ml})$, and drying over anhydrous sodium sulfate, and removal of the solvent in vacuo gave the crude product, which was purified on Combiflash using ethylacetate: dichloromethane $(2: 3 \mathrm{v} / \mathrm{v})$ as an eluent. The resultant Ylide product afforded in fair to good yields.

Benzoyl(isoquinolin-2-ium-2-yl)amide (5a): Yield 55\%, $\mathrm{mp}$ 185.2-188.6 ${ }^{\circ} \mathrm{C}$; ${ }^{1} \mathrm{HNMR}\left(\mathrm{CDCl}_{3}\right) \delta(\mathrm{ppm}):$ 7.55-7.64 (m, 3H, $\mathrm{C}_{3}, \mathrm{C}_{4}$, $\left.\mathrm{C}_{5^{\prime}}-\mathrm{H}\right), 8.20\left(\mathrm{~d}, 2 \mathrm{H}, \mathrm{J}=3.0 \mathrm{~Hz}, \mathrm{C}_{2^{\prime}}, \mathrm{C}_{6}-\mathrm{H}\right), 8.31\left(\mathrm{~d}, 1 \mathrm{H}, J=6.9 \mathrm{~Hz}, \mathrm{C}_{4}-\mathrm{H}\right)$, 8.38-8.44 (m, $\left.4 \mathrm{H}, \mathrm{C}_{6}, \mathrm{C}_{7}, \mathrm{C}_{8}, \mathrm{C}_{9}-\mathrm{H}\right), 8.67\left(\mathrm{~d}, 1 \mathrm{H}, J=6.9 \mathrm{~Hz}, \mathrm{C}_{3}-\mathrm{H}\right), 9.93$ $\left(\mathrm{s}, 1 \mathrm{H}, \mathrm{C}_{1}-\mathrm{H}\right)$.

Isoquinolin-2-ium-2-yl(4-methylbenzoyl)amide (5b): Yield 60\%, mp 174.3-175.8; ${ }^{1} \mathrm{HNMR}\left(\mathrm{CDCl}_{3}\right) \delta$ (ppm): $2.38\left(\mathrm{~s}, 3 \mathrm{H}, \mathrm{CH}_{3}\right.$ group), $7.28\left(\mathrm{~d}, 2 \mathrm{H}, \mathrm{J}=8.1 \mathrm{~Hz}, \mathrm{C}_{3}, \mathrm{C}_{5}, \mathrm{H}\right), 7.91\left(\mathrm{dd}, 2 \mathrm{H}, J=1.8,6.3 \mathrm{~Hz}, \mathrm{C}_{7}, \mathrm{C}_{8}-\mathrm{H}\right)$, $7.97\left(\mathrm{dd}, 1 \mathrm{H}, J=7.8,7.2 \mathrm{~Hz}, \mathrm{C}_{9}-\mathrm{H}\right), 8.14\left(\mathrm{dd}, 1 \mathrm{H}=2.1,7.5 \mathrm{~Hz}, \mathrm{C}_{4}-\mathrm{H}\right)$, $8.25\left(\mathrm{~d}, 1 \mathrm{H}, J=8.4 \mathrm{~Hz}, \mathrm{C}_{6}-\mathrm{H}\right), 8.41\left(\mathrm{dd}, 2 \mathrm{H}, \mathrm{J}=6.9,5.4 \mathrm{~Hz}, \mathrm{C}_{2^{\prime}}, \mathrm{C}_{6^{\prime}}-\mathrm{H}\right), 8.56$ (dd, $\left.1 \% J=2.1,4.8 \mathrm{~Hz}, \mathrm{C}_{3}-\mathrm{H}\right), 9.89\left(\mathrm{~s}, 1 \mathrm{H}, \mathrm{C}_{1}-\mathrm{H}\right)$.

Isoquinolin-2-ium-2-yl(4-methoxybenzoyl)amide (5c):Yield 58\%; mp 169.5-171.2 ${ }^{\circ} \mathrm{C} ;{ }^{1} \mathrm{HNMR}\left(\mathrm{CDCl}_{3}\right) \delta$ (ppm): 3.85 (s, $3 \mathrm{H}, \mathrm{OCH}_{3}$ group), $7.16\left(\mathrm{~d}, 2 \mathrm{H}, J=8.1 \mathrm{~Hz}, \mathrm{C}_{3}, \mathrm{C}_{5},-\mathrm{H}, 7.72(\mathrm{dd}, 2 \mathrm{H}, J=1.8,6.3 \mathrm{~Hz}\right.$, $\left.\mathrm{C}_{7}, \mathrm{C}_{8}-\mathrm{H}\right), 7.99$ (dd, $\left.2 \mathrm{H}, J=6.9,5.4 \mathrm{~Hz}, \mathrm{C}_{2}, \mathrm{C}_{6}, \mathrm{H}\right), 8.10$ (dd, $1 \mathrm{H}, J=7.8$, $\left.7.2 \mathrm{~Hz}, \mathrm{C}_{9}-\mathrm{H}\right), 8.28\left(\mathrm{dd}, 1 \mathrm{H}, \mathrm{J}=2.1,8.1 \mathrm{~Hz}, \mathrm{C}_{4}-\mathrm{H}\right), 8.58(\mathrm{~d}, 1 \mathrm{H}, J=8.1 \mathrm{~Hz}$, $\left.\mathrm{C}_{6}-\mathrm{H}\right), 8.71\left(\mathrm{dd}, 1 \mathrm{H}, J=2.1,6.9 \mathrm{~Hz}, \mathrm{C}_{3}-\mathrm{H}\right), 10.10\left(\mathrm{~s}, 1 \mathrm{H}, \mathrm{C}_{1}-\mathrm{H}\right)$.

(4-Ethylbenzoyl)(isoquinolin-2-ium-2yl)amide (5d): Yield 58\%; mp 173.4-174. $5^{\circ} \mathrm{C} ;{ }^{1} \mathrm{HNMR}\left(\mathrm{CDCl}_{3}\right) \delta(\mathrm{ppm}): 1.25(\mathrm{t}, 3 \mathrm{H}, \mathrm{J}=7.5 \mathrm{~Hz}$, $\left.-\mathrm{CH}_{2}-\mathrm{CH}_{3}\right), 2.66-2.73\left(\mathrm{q}, 2 \mathrm{H}, \mathrm{J}=7.2 \mathrm{~Hz},-\mathrm{CH}_{2}-\mathrm{CH}_{3}\right), 7.29$ (d, $1 \mathrm{H}, \mathrm{J}=8.4$ $\left.\mathrm{Hz}, \mathrm{C}_{6}-\mathrm{H}\right) 7.79-7.85(\mathrm{~m}, 1 \mathrm{H}, \mathrm{C} 7-\mathrm{H}), 7.93-8.03\left(\mathrm{~m}, 4 \mathrm{H}, \mathrm{C}_{5}, \mathrm{C}_{8}, \mathrm{C}_{3}, \mathrm{C}_{5}, \mathrm{H}\right)$, 8.12-8.17 (m, 3H, $\left.\mathrm{C}_{4}, \mathrm{C}_{2}, \mathrm{C}_{6}-\mathrm{H}\right), 8.46\left(\mathrm{dd}, 1 \mathrm{H}, \mathrm{J}=1.2,5.7 \mathrm{~Hz}, \mathrm{C}_{3}-\mathrm{H}\right), 9.87$ (s, $\left.1 \mathrm{H}, \mathrm{C}_{1}-\mathrm{H}\right)$.

Isoquinolin-2-ium-2-yl-(4-propylbenzoyl)amide (5e): Yield 53\%; mp 164.2-165.7 ${ }^{\circ} \mathrm{C} ;{ }^{1} \mathrm{HNMR}\left(\mathrm{CDCl}_{3}\right) \delta(\mathrm{ppm}): 0.94(\mathrm{t}, 3 \mathrm{H}, \mathrm{J}=7.5 \mathrm{~Hz}$, $\left.-\mathrm{CH}_{2}-\mathrm{CH}_{2}-\mathrm{CH}_{3}\right), 1.61-1.71\left(\mathrm{~m}, 2 \mathrm{H},-\mathrm{CH}_{2}-\mathrm{CH}_{2}-\mathrm{CH}_{3}\right), 2.64(\mathrm{t}, 2 \mathrm{H}, \mathrm{J}=8.1$ $\left.\mathrm{Hz},-\mathrm{CH}_{2}-\mathrm{CH}_{2}-\mathrm{CH}_{3}\right), 7.24\left(\mathrm{~d}, 1 \mathrm{H}, \mathrm{J}=8.4 \mathrm{~Hz}, \mathrm{C}_{6}-\mathrm{H}\right) 7.79-7.85\left(\mathrm{~m}, 1 \mathrm{H}, \mathrm{C}_{7}-\right.$ H), 7.91-7.94 (m, 2H, C $\left.{ }_{5}, \mathrm{C}_{8}-\mathrm{H}\right), 7.99\left(\mathrm{~d}, 2 \mathrm{H}, \mathrm{J}=6.9 \mathrm{~Hz}, \mathrm{C}_{3}, \mathrm{C}_{5}, \mathrm{H}\right), 8.11-$ $8.15\left(\mathrm{~m}, 3 \mathrm{H}, \mathrm{C}_{4}, \mathrm{C}_{2}, \mathrm{C}_{6}-\mathrm{H}\right), 8.45\left(\mathrm{dd}, 1 \mathrm{H}, \mathrm{J}=1.5,5.7 \mathrm{~Hz}, \mathrm{C}_{3}-\mathrm{H}\right), 9.87(\mathrm{~s}$, $\left.1 \mathrm{H}, \mathrm{C}_{1}-\mathrm{H}\right)$.

(4-Butylbenzoylimino)(isoquinolin2-ium-2-yl)amide (5f): Yield 58\%; mp 154.7-156 ${ }^{\circ} \mathrm{C} ;{ }^{1} \mathrm{HNMR}\left(\mathrm{CDCl}_{3}\right) \delta(\mathrm{ppm}): 0.93(\mathrm{t}, 3 \mathrm{H}, \mathrm{J}=6.9 \mathrm{~Hz}$, $\left.-\mathrm{CH}_{2}-\mathrm{CH}_{2}-\mathrm{CH}_{2}-\mathrm{CH}_{3}\right), 1.36\left(\mathrm{q}, 2 \mathrm{H}, \mathrm{J}=7.5 \mathrm{~Hz},-\mathrm{CH}_{2}-\mathrm{CH}_{2}-\mathrm{CH}_{2}-\mathrm{CH}_{3}\right) 1.62$ $\left(\mathrm{m}, 2 \mathrm{H},-\mathrm{CH}_{2}-\mathrm{CH}_{2}-\mathrm{CH}_{2}-\mathrm{CH}_{3}\right), 2.66\left(\mathrm{t}, 2 \mathrm{H}, \mathrm{J}=7.8, \mathrm{~Hz},-\mathrm{CH}_{2}-\mathrm{CH}_{2}-\mathrm{CH}_{2}-\right.$ $\left.\mathrm{CH}_{3}\right), 7.25\left(\mathrm{~d}, 2 \mathrm{H}, \mathrm{J}=6.0 \mathrm{~Hz}, \mathrm{C}_{3}, \mathrm{C}_{5}, \mathrm{H}\right), 7.79-7.85\left(\mathrm{~m}, 1 \mathrm{H}, \mathrm{C}_{7}-\mathrm{H}\right), 7.91-$ $7.96\left(\mathrm{~m}, 1 \mathrm{H}, \mathrm{C}_{8}-\mathrm{H}\right), 7.96\left(\mathrm{~d}, 2 \mathrm{H}, \mathrm{J}=8.1 \mathrm{~Hz}, \mathrm{C}_{2}, \mathrm{C}_{6}-\mathrm{H}\right), 8.09-8.16(\mathrm{~m}, 3 \mathrm{H}$, $\left.\mathrm{C}_{4}, \mathrm{C}_{5}, \mathrm{C}_{6}-\mathrm{H}\right), 8.45\left(\mathrm{dd}, 1 \mathrm{H}, \mathrm{J}=1.5,5.7 \mathrm{~Hz}, \mathrm{C}_{3}-\mathrm{H}\right), 9.88\left(\mathrm{~s}, 1 \mathrm{H}, \mathrm{C}_{1}-\mathrm{H}\right)$.

(4-(tert-butyl)benzoyl)(isoquinolin-2-ium-2-yl)amide (5g): Yield 65.5\%; mp 172.2-173.5 ${ }^{\circ} \mathrm{C}$; ${ }^{1} \mathrm{HNMR}\left(\mathrm{CDCl}_{3}\right) \delta(\mathrm{ppm}): 1.36(\mathrm{~s}, 9 \mathrm{H}$, $t$-butyl group), $7.45\left(\mathrm{dd}, 2 \mathrm{H}, \mathrm{J}=2.1,8.7 \mathrm{~Hz}, \mathrm{C}_{6}, \mathrm{C}_{7}-\mathrm{H}\right), 7.77-7.83(\mathrm{~m}$, $\left.1 \mathrm{H}, \mathrm{C}_{5}-\mathrm{H}\right), 7.87-7.00\left(\mathrm{~m}, 3 \mathrm{H}, \mathrm{C}_{8}\right.$ and $\left.\mathrm{C}_{3}, \mathrm{C}_{5}-\mathrm{H}\right), 8.07(\mathrm{~d}, 1 \mathrm{H}, \mathrm{J}=8.1 \mathrm{~Hz}$, $\left.\mathrm{C}_{4}-\mathrm{H}\right), 8.13\left(\mathrm{~d}, 2 \mathrm{H}, \mathrm{J}=8.7 \mathrm{~Hz}, \mathrm{C}_{2}, \mathrm{C}_{6}-\mathrm{H}\right), 8.43(\mathrm{dd}, 1 \mathrm{H}, \mathrm{J}=1.2,5.7 \mathrm{~Hz}$, $\left.\mathrm{C}_{3}-\mathrm{H}\right), 9.91\left(\mathrm{~s}, 1 \mathrm{H}, \mathrm{C}_{1}-\mathrm{H}\right)$.

(4-Fluorobenzoyl)(isoquinolin-2-ium-2-yl)amide (5h): Yield 49.5\%; mp 183.1-184.8 ${ }^{\circ} \mathrm{C}$; ${ }^{1} \mathrm{HNMR}\left(\mathrm{CDCl}_{3}\right) \delta(\mathrm{ppm})$ : 7.35-7.45 (m, $2 \mathrm{H}$, $\left.\mathrm{C}_{6}, \mathrm{C}_{7}-\mathrm{H}\right), 7.75$ ( dd, $\left.2 \mathrm{H}, \mathrm{J}=1.5,6.6 \mathrm{~Hz}, \mathrm{C}_{3}, \mathrm{C}_{5}, \mathrm{H}\right), 7.83-7.89\left(\mathrm{~m}, 1 \mathrm{H}, \mathrm{C}_{5}-\right.$ $\mathrm{H})$, 7.97-8.17 (m, $\left.4 \mathrm{H}, \mathrm{C}_{4}, \mathrm{C}_{8}, \mathrm{C}_{2}, \mathrm{C}_{6}-\mathrm{H}\right), 8.47\left(\mathrm{dd}, 1 \mathrm{H}, \mathrm{J}=6.3 \mathrm{~Hz}, \mathrm{C}_{3}-\mathrm{H}\right)$, $8.56\left(\mathrm{dd}, 1 \mathrm{H}, \mathrm{J}=6.3 \mathrm{~Hz}, \mathrm{C}_{3}-\mathrm{H}\right), 9.74\left(\mathrm{~s}, 1 \mathrm{H}, \mathrm{C}_{1}-\mathrm{H}\right)$. 
Citation: Gangapuram M, Eyunni S, Redda KK (2014) Synthesis and Pharmacological Evolution of Tetrahydroisoquinolines as Anti Breast Cancer Agents. J Cancer Sci Ther 6: 161-169. doi:10.4172/1948-5956.1000266

(4-Chlorobenzoyl)(isoquinolin-2-ium-2-yl)amide (5i): Yield 70\%; mp 180-182.4 ${ }^{\circ} \mathrm{C} ;{ }^{1} \mathrm{HNMR}\left(\mathrm{CDCl}_{3}\right) \delta(\mathrm{pprn}): 7.70\left(\mathrm{~d}, 2 \mathrm{H}, J=8.1 \mathrm{~Hz}, \mathrm{C}_{3}\right.$, $\left.\mathrm{C}_{5^{\prime}}-\mathrm{H}\right), 7.72\left(\mathrm{dd}, 2 \mathrm{H}, J=1.8,6.3 \mathrm{~Hz}, \mathrm{C}_{6}-\mathrm{H}\right), 8.01-8.09\left(\mathrm{~m}, 3 \mathrm{H}, \mathrm{C}_{4}, \mathrm{C}_{7}, \mathrm{C}_{8}-\right.$ $\mathrm{H}), 8.14\left(\mathrm{dd}, 2 \mathrm{H}, J=6.9,5.4 \mathrm{~Hz}, \mathrm{C}_{2}, \mathrm{C}_{6}-\mathrm{H}\right), 8.35\left(\mathrm{~d}, 1 \mathrm{H}, J=8.1 \mathrm{~Hz}, \mathrm{C}_{5}-\mathrm{H}\right)$, $9.08\left(\mathrm{dd}, 1 \mathrm{H}, J=2.1,6.9 \mathrm{~Hz}, \mathrm{C}_{3}-\mathrm{H}\right), 10.10\left(\mathrm{~s}, 1 \mathrm{H}, \mathrm{C}_{1}-\mathrm{H}\right)$.

(4-Bromobenzoyl)(isoquinolin-2-ium-2-yl)amide (5j): Yield 58.6\%; mp 189.2-190.1 ${ }^{\circ} \mathrm{C} ;{ }^{1} \mathrm{HNMR}\left(\mathrm{CDCl}_{3}\right) \delta(\mathrm{ppm}):$ 7.38-7.47 (m, $2 \mathrm{H}$, $\left.\mathrm{C}_{6}, \mathrm{C}_{7}-\mathrm{H}\right), 7.78\left(\mathrm{dd}, 2 \mathrm{H}, \mathrm{J}=1.2,6.7 \mathrm{~Hz}, \mathrm{C}_{3}, \mathrm{C}_{5},-\mathrm{H}\right), 7.84-7.91\left(\mathrm{~m}, 1 \mathrm{H}, \mathrm{C}_{5}-\right.$ $\mathrm{H}), 7.99-8.17\left(\mathrm{~m}, 4 \mathrm{H}, \mathrm{C}_{4}, \mathrm{C}_{8}, \mathrm{C}_{2}, \mathrm{C}_{6}-\mathrm{H}\right), 8.24(\mathrm{dd}, 1 \mathrm{H}, \mathrm{J}=1.2,8.7 \mathrm{~Hz}$, $\left.\mathrm{C}_{3}-\mathrm{H}\right), 9.39\left(\mathrm{~s}, 1 \mathrm{H}, \mathrm{C}_{1}-\mathrm{H}\right)$.

Isoquinolin-2-ium-2-yl[4-(trifluoromethyl)benzoyl]amide (5k): Yield 68.4\%; mp 192.3-193.1 ${ }^{\circ} \mathrm{C} ;{ }^{1} \mathrm{HNMR}\left(\mathrm{CDCl}_{3}\right) \delta(\mathrm{ppm}): 7.69(\mathrm{~d}, 2 \mathrm{H}$, $\left.\mathrm{J}=8.1 \mathrm{~Hz}, \mathrm{C}_{3}, \mathrm{C}_{5}, \mathrm{H}\right), 7.82\left(\mathrm{dtd}, 1 \mathrm{H}, \mathrm{J}=1.2,1.5,0.9 \mathrm{~Hz}, \mathrm{C}_{7}-\mathrm{H}\right), 7.89-8.01$ $\left(\mathrm{m}, 3 \mathrm{H}, \mathrm{C}_{5}, \mathrm{C}_{6}, \mathrm{C}_{8}-\mathrm{H}\right), 8.08\left(\mathrm{~d}, 1 \mathrm{H}, \mathrm{J}=7.8 \mathrm{~Hz}, \mathrm{C}_{4}-\mathrm{H}\right), 8.31(\mathrm{~d}, 2 \mathrm{H}, \mathrm{J}=8.1$ $\left.\mathrm{Hz}, \mathrm{C}_{2}, \mathrm{C}_{6}-\mathrm{H}\right), 8.43$ (dd, $\left.1 \mathrm{H}, \mathrm{J}=1.2,5.7 \mathrm{~Hz}, \mathrm{C}_{3}-\mathrm{H}\right), 9.92\left(\mathrm{~s}, 1 \mathrm{H}, \mathrm{C}_{1}-\mathrm{H}\right)$.

Isoquinolin-2-ium-2-yl(3,4,5-trimethoxybenzoyl)amide (5l): Yield 62.5\%; mp 188.9-191.3 ${ }^{\circ} \mathrm{C} ;{ }^{1} \mathrm{HNMR}\left(\mathrm{CDCl}_{3}\right) \delta$ (ppm): $3.90(\mathrm{~s}, 3 \mathrm{H}$, p- $\mathrm{CH}_{3}$ group), 3.95 (s, $6 \mathrm{H}, \mathrm{m}-\mathrm{CH}_{3}$ group), $7.52\left(\mathrm{~s}, 2 \mathrm{H}, \mathrm{C}_{2}, \mathrm{C}_{6}-\mathrm{H}\right), 7.82$ (dd, $\left.1 \mathrm{H}, \mathrm{J}=6.9,8.1 \mathrm{~Hz}, \mathrm{C}_{7}-\mathrm{H}\right), 7.89-8.01\left(\mathrm{~m}, 3 \mathrm{H}, \mathrm{C}_{5}, \mathrm{C}_{6}, \mathrm{C}_{8}-\mathrm{H}\right), 8.08(\mathrm{~d}$, $\left.1 \mathrm{H}, \mathrm{J}=8.1 \mathrm{~Hz}, \mathrm{C}_{4}-\mathrm{H}\right), 8.43\left(\mathrm{dd}, 1 \mathrm{H}, \mathrm{J}=1.5,5.7 \mathrm{~Hz}, \mathrm{C}_{3}-\mathrm{H}\right), 9.84(\mathrm{~s}, 1 \mathrm{H}$, $\left.\mathrm{C}_{1}-\mathrm{H}\right)$.

Isoquinolin-2-ium-2-yl(4-nitrobenzoyl)amide (5m): Yield 58\%; mp 173.1-174.5 ${ }^{\circ} \mathrm{C} ;{ }^{1} \mathrm{HNMR}\left(\mathrm{CDCl}_{3}\right) \delta(\mathrm{ppm}): 7.49(\mathrm{~d}, 2 \mathrm{H}, \mathrm{J}=8.1 \mathrm{~Hz}$, $\left.\mathrm{C}_{3}, \mathrm{C}_{5}, \mathrm{H}\right), 7.79-7.83\left(\mathrm{~m} \mathrm{1H}, \mathrm{C}_{7}-\mathrm{H}\right), 7.89-8.03\left(\mathrm{~m}, 3 \mathrm{H}, \mathrm{C}_{5}, \mathrm{C}_{6}, \mathrm{C}_{8}-\mathrm{H}\right)$, $8.09\left(\mathrm{~d}, 1 \mathrm{H}, \mathrm{J}=7.5 \mathrm{~Hz}, \mathrm{C}_{4}-\mathrm{H}\right), 8.34\left(\mathrm{~d}, 2 \mathrm{H}, \mathrm{J}=8.1 \mathrm{~Hz}, \mathrm{C}_{2}, \mathrm{C}_{6}-\mathrm{H}\right), 8.45$ (dd, $\left.1 \mathrm{H}, \mathrm{J}=1.2,5.7 \mathrm{~Hz}, \mathrm{C}_{3}-\mathrm{H}\right), 9.91\left(\mathrm{~s}, 1 \mathrm{H}, \mathrm{C}_{1}-\mathrm{H}\right)$.

Isoquinolin-2-ium-2-yl(phenylsulfonyl)amide (5n): Yield 51.5\%; mp 203.5-204.6 ${ }^{\circ} \mathrm{C} ;{ }^{1} \mathrm{HNMR}\left(\mathrm{CDCl}_{3}\right) \delta$ (ppm): 7.33-7.42 (m, 3H, $\mathrm{C}_{3}$, $\left.\mathrm{C}_{4}, \mathrm{C}_{5}, \mathrm{H}\right), 7.76\left(\mathrm{dd}, 2 \mathrm{H}, \mathrm{J}=1.5,6.9 \mathrm{~Hz}, \mathrm{C}_{6}, \mathrm{C}_{7}-\mathrm{H}\right), 7.83(\mathrm{~d}, 2 \mathrm{H}, \mathrm{J}=8.1 \mathrm{~Hz}$, $\left.\mathrm{C}_{2}, \mathrm{C}_{6}-\mathrm{H}\right), 7.95\left(\mathrm{dd}, 2 \mathrm{H}, \mathrm{J}=1.2,6.0 \mathrm{~Hz}, \mathrm{C}_{5}, \mathrm{C}_{8}-\mathrm{H}\right), 8.04(\mathrm{~d}, 1 \mathrm{H}, \mathrm{J}=8.1 \mathrm{~Hz}$, $\left.\mathrm{C}_{4}-\mathrm{H}\right), 8.09(\mathrm{dd}, 1 \mathrm{H}, \mathrm{J}=1.5,5.7 \mathrm{~Hz}, \mathrm{C} 3-\mathrm{H}), 9.25\left(\mathrm{~s}, 1 \mathrm{H}, \mathrm{C}_{1}-\mathrm{H}\right)$.

Isoquinolin-2-ium-2-yl((4-methylphenyl)sulfonyl)amide (5o): Yield 56.8\%; mp 210.6-211.8 ${ }^{\circ} \mathrm{C}$; ${ }^{1} \mathrm{HNMR}\left(\mathrm{CDCl}_{3}\right) \delta(\mathrm{ppm}): 2.35(\mathrm{~s}, 3 \mathrm{H}$, $\mathrm{CH}_{3}$ group), $7.14\left(\mathrm{~d}, 2 \mathrm{H}, \mathrm{J}=7.8 \mathrm{~Hz}, \mathrm{C}_{3}, \mathrm{C}_{5}, \mathrm{H}\right), 7.64(\mathrm{~d}, 2 \mathrm{H}, \mathrm{J}=8.4 \mathrm{~Hz}$, $\left.\mathrm{C}_{6}, \mathrm{C}_{7}-\mathrm{H}\right), 7.84\left(\mathrm{~d}, 2 \mathrm{H}, J=7.5 \mathrm{~Hz}, \mathrm{C}_{5}, \mathrm{C}_{8}-\mathrm{H}\right), 7.95(\mathrm{~d}, 2 \mathrm{H}, J=6.3 \mathrm{~Hz}$, $\left.\mathrm{C}_{2}, \mathrm{C}_{6}-\mathrm{H}\right), 8.05\left(\mathrm{~d}, 1 \mathrm{H}, J=8.4, \mathrm{~Hz}, \mathrm{C}_{4}-\mathrm{H}\right), 8.11(\mathrm{dd}, 1 \mathrm{H}, J=1.8,5.4 \mathrm{~Hz}$, $\left.\mathrm{C}_{3}-\mathrm{H}\right), 9.27\left(\mathrm{~s}, 1 \mathrm{H}, \mathrm{C}_{1}-\mathrm{H}\right)$.

Isoquinolin-2-ium-2-yl((4-methoxyphenyl)sulfonyl)amide (5p): Yield 55\%; mp 220.3-221.5 ${ }^{\circ}$; ${ }^{1} \mathrm{HNMR}\left(\mathrm{CDCl}_{3}\right) \delta(\mathrm{ppm}): 3.83$ (s, 3H, $\mathrm{OCH}_{3}$ group), $7.12\left(\mathrm{~d}, 2 \mathrm{H}, J=7.9 \mathrm{~Hz}, \mathrm{C}_{3^{\prime}}, \mathrm{C}_{5}, \mathrm{H}\right), 7.99(\mathrm{dd}, 2 \mathrm{H}, J=8.1,5.4$ $\left.\mathrm{Hz}, \mathrm{C}_{2}, \mathrm{C}_{6}-\mathrm{H}\right), 7.69\left(\mathrm{dd}, 1 \mathrm{H}, J=1.8,6.3 \mathrm{~Hz}, \mathrm{C}_{8}-\mathrm{H}\right), 8.10-8.14\left(\mathrm{rn}, 2 \mathrm{H}, \mathrm{C}_{7}\right.$, $\left.\mathrm{C}_{6}-\mathrm{H}\right), 8.15\left(\mathrm{dd}, 1 \mathrm{H}, \mathrm{J}=7.8,7.2 \mathrm{~Hz}, \mathrm{C}_{9}-\mathrm{H}\right), 8.25(\mathrm{dd}, 1 \mathrm{H}, J=2.1,8.1 \mathrm{~Hz}$, $\left.\mathrm{C}_{4}-\mathrm{H}\right), 8.85\left(\mathrm{dd}, 1 \mathrm{H}, \mathrm{J}=2.1,6.9 \mathrm{~Hz}, \mathrm{C}_{3}-\mathrm{H}\right), 10.45\left(\mathrm{~s}, 1 \mathrm{H}, \mathrm{C}_{1}-\mathrm{H}\right)$.

((4-Ethylphenyl)sulfonyl)(isoquinolin-2-ium-2-yl)amide (5q): Yield 64.3\%; mp 247.6-248.6 ${ }^{\circ} \mathrm{C} ;{ }^{1} \mathrm{HNMR}\left(\mathrm{CDCl}_{3}\right) \delta(\mathrm{ppm}): 1.21(\mathrm{t}, 3 \mathrm{H}$, $\left.\mathrm{J}=7.8 \mathrm{~Hz},-\mathrm{CH}_{2}-\mathrm{CH}_{3}\right), 2.61-2.68\left(\mathrm{q}, 2 \mathrm{H}, \mathrm{J}=7.5 \mathrm{~Hz},-\mathrm{CH}_{2}-\mathrm{CH}_{3}\right), 7.17(\mathrm{~d}$, $\left.2 \mathrm{H}, \mathrm{J}=7.8 \mathrm{~Hz}, \mathrm{C}_{3}, \mathrm{C}_{5}, \mathrm{H}\right), 7.68\left(\mathrm{~d}, 2 \mathrm{H}, J=8.1 \mathrm{~Hz}, \mathrm{C}_{6}, \mathrm{C}_{7}-\mathrm{H}\right), 7.79-7.85(\mathrm{~m}$, $\left.2 \mathrm{H}, \mathrm{C}_{5}, \mathrm{C}_{8}-\mathrm{H}\right), 7.93\left(\mathrm{~d}, 2 \mathrm{H}, J=6.3 \mathrm{~Hz}, \mathrm{C}_{2}, \mathrm{C}_{6}-\mathrm{H}\right), 8.03(\mathrm{dd}, 1 \mathrm{H}, J=0.6,7.5$ $\left.\mathrm{Hz}, \mathrm{C}_{4}-\mathrm{H}\right), 8.13$ (dd, $\left.1 \mathrm{H}, J=1.8,5.1 \mathrm{~Hz}, \mathrm{C}_{3}-\mathrm{H}\right), 9.27\left(\mathrm{~s}, 1 \mathrm{H}, \mathrm{C}_{1}-\mathrm{H}\right)$.

((4-Butylphenyl)sulfonyl)(isoquinolin-2-ium-2-yl)amdie (5r): Yield 53.9\%; mp 225.9-226.9 ${ }^{\circ} \mathrm{C} ;{ }^{1} \mathrm{HNMR}\left(\mathrm{CDCl}_{3}\right) \delta(\mathrm{ppm}): 0.90(\mathrm{t}, 3 \mathrm{H}$, $\left.\mathrm{J}=7.5 \mathrm{~Hz},-\mathrm{CH}_{2}-\mathrm{CH}_{2}-\mathrm{CH}_{2}-\mathrm{CH}_{3}\right), 1.29\left(\mathrm{q}, 2 \mathrm{H}, \mathrm{J}=7.5 \mathrm{~Hz},-\mathrm{CH}_{2}-\mathrm{CH}_{2}-\mathrm{CH}_{2}-\right.$ $\left.\mathrm{CH}_{3}\right), 1.51-1.61\left(\mathrm{~m}, 2 \mathrm{H},-\mathrm{CH}_{2}-\mathrm{CH}_{2}-\mathrm{CH}_{2}-\mathrm{CH}_{3}\right), 2.60(\mathrm{t}, 2 \mathrm{H}, \mathrm{J}=8.1, \mathrm{~Hz}$, $\left.-\mathrm{CH}_{2}-\mathrm{CH}_{2}-\mathrm{CH}_{2}-\mathrm{CH}_{3}\right), 7.15\left(\mathrm{~d}, 2 \mathrm{H}, \mathrm{J}=6.0 \mathrm{~Hz}, \mathrm{C}_{3}, \mathrm{C}_{5}-\mathrm{H}\right), 7.65-7.68(\mathrm{~m}$, $\left.2 \mathrm{H}, \mathrm{C}_{6}, \mathrm{C}_{7}-\mathrm{H}\right), 7.79-7.85\left(\mathrm{~m}, 2 \mathrm{H}, \mathrm{C}_{5}, \mathrm{C}_{8}-\mathrm{H}\right), 7.94\left(\mathrm{~d}, 2 \mathrm{H}, \mathrm{J}=8.1 \mathrm{~Hz}, \mathrm{C}_{2}\right.$,
$\left.\mathrm{C}_{6}-\mathrm{H}\right), 8.04\left(\mathrm{~d}, 1 \mathrm{H}, \mathrm{J}=8.4 \mathrm{~Hz}, \mathrm{C}_{4}-\mathrm{H}\right), \quad, 8.13\left(\mathrm{dd}, 1 \mathrm{H}, \mathrm{J}=1.5,5.7 \mathrm{~Hz}, \mathrm{C}_{3}-\right.$ $\mathrm{H}), 9.26\left(\mathrm{~s}, 1 \mathrm{H}, \mathrm{C}_{1}-\mathrm{H}\right)$.

(Furan-2-carbonyl)(isoquinolin-2-ium-2-yl)amide (5s): Yield 65.8\%; mp 147.5-148.9 ${ }^{\circ} \mathrm{C}^{1} \mathrm{HNMR}\left(\mathrm{CDCl}_{3}\right) \delta(\mathrm{ppm}): 6.51$ (d, $1 \mathrm{H}, \mathrm{J}=1.5$ $\left.\mathrm{Hz}, \mathrm{C}_{4},-\mathrm{H}\right), 7.02\left(\mathrm{~d}, 1 \mathrm{H}, \mathrm{J}=6.3 \mathrm{~Hz}, \mathrm{C}_{5}-\mathrm{H}\right), 7.17-7.20\left(\mathrm{~m}, 3 \mathrm{H}, \mathrm{C}_{6}, \mathrm{C}_{7}, \mathrm{C}_{8}-\mathrm{H}\right)$, $7.43\left(\mathrm{~s}, 1 \mathrm{H}, \mathrm{C}_{3}, \mathrm{H}\right), 7.64\left(\mathrm{~s}, 1 \mathrm{H}, \mathrm{C}_{5},-\mathrm{H}\right), 8.01\left(\mathrm{~d}, 1 \mathrm{H}, \mathrm{J}=8.4 \mathrm{~Hz}, \mathrm{C}_{4}-\mathrm{H}\right)$, , $8.10\left(\mathrm{~d}, 1 \mathrm{H}, \mathrm{J}=5.7 \mathrm{~Hz}, \mathrm{C}_{3}-\mathrm{H}\right), 9.21\left(\mathrm{~s}, 1 \mathrm{H}, \mathrm{C}_{1}-\mathrm{H}\right)$.

(6-Chloronicotinoyl)(isoquinolin-2-ium-2-yl)amide (5t): Yield 58\%; mp 154.5-156.7 ${ }^{\circ} \mathrm{C} ;{ }^{1} \mathrm{HNMR}\left(\mathrm{CDCl}_{3}\right) \delta$ (ppm): 7.37 (d, $1 \mathrm{H}, \mathrm{J}=7.5$ $\left.\mathrm{Hz}, \mathrm{C}_{4}-\mathrm{H}\right), 7.83-7.88\left(\mathrm{~m}, 1 \mathrm{H}, \mathrm{C}_{5}-\mathrm{H}\right), 7.39-7.04\left(\mathrm{~m}, 3 \mathrm{H}, \mathrm{C}_{6}, \mathrm{C}_{7}, \mathrm{C}_{8}-\mathrm{H}\right)$, $8.13\left(\mathrm{~d}, 1 \mathrm{H}, \mathrm{J}=9.60 \mathrm{~Hz}, \mathrm{C}_{4}-\mathrm{H}\right), 8.42-8.47\left(\mathrm{~m}, 2 \mathrm{H}, \mathrm{C}_{3}, \mathrm{C}_{5},-\mathrm{H}\right), 9.19(\mathrm{~d}, 1 \mathrm{H}$, $\left.\mathrm{J}=1.8 \mathrm{~Hz}, \mathrm{C}_{2}, \mathrm{H}\right), 9.90\left(\mathrm{~s}, 1 \mathrm{H}, \mathrm{C}_{1}-\mathrm{H}\right)$.

(2-Chloro-6-methylisonicotinoyl)(isoquinolin-2-ium-2-yl)amide (5u): Yield 57.8\%; mp 196.7-197.8 ${ }^{\circ} \mathrm{C} ;{ }^{1} \mathrm{HNMR}\left(\mathrm{CDCl}_{3}\right) \delta$ (ppm): 2.55 $\left(\mathrm{s}, 3 \mathrm{H},-\mathrm{CH}_{3}\right), 7.03\left(\mathrm{~d}, 1 \mathrm{H}, \mathrm{J}=6.3 \mathrm{~Hz}, \mathrm{C}_{7}-\mathrm{H}\right), 7.16-7.20\left(\mathrm{~m}, 3 \mathrm{H}, \mathrm{C}_{5}, \mathrm{C}_{6}\right.$, $\left.\mathrm{C}_{8}-\mathrm{H}\right), 7.44\left(\mathrm{~d}, 2 \mathrm{H}, \mathrm{J}=7.8 \mathrm{~Hz}, \mathrm{C}_{3}, \mathrm{C}_{5}, \mathrm{H}\right), 8.09\left(\mathrm{~d}, 1 \mathrm{H}, \mathrm{J}=8.1 \mathrm{~Hz}, \mathrm{C}_{4}-\mathrm{H}\right)$, $8.45\left(\mathrm{dd}, 1 \mathrm{H}, \mathrm{J}=1.5,5.7 \mathrm{~Hz}, \mathrm{C}_{3}-\mathrm{H}\right), 9.84\left(\mathrm{~s}, 1 \mathrm{H}, \mathrm{C}_{1}-\mathrm{H}\right)$.

(Benzo[d]thiazole-2-carbonyl)(isoquinolin-2-ium-2-yl)amide (5v): Yield 55.4\%; mp 225.9-226.9 ${ }^{\circ} \mathrm{C} ;{ }^{1} \mathrm{HNMR}\left(\mathrm{CDCl}_{3}\right) \delta$ (ppm): 7.32 $7.34\left(\mathrm{~m}, 1 \mathrm{H}, \mathrm{C}_{8}-\mathrm{H}\right), 7.47-7.52\left(\mathrm{~m}, 1 \mathrm{H}, \mathrm{C}_{7}, \mathrm{H}\right), 8.01-8.09\left(\mathrm{~m}, 2 \mathrm{H}, \mathrm{C}_{6}\right.$, $\left.\mathrm{C}_{8}-\mathrm{H}\right), 8.10-8.15\left(\mathrm{~m}, 2 \mathrm{H}, \mathrm{C}_{4}, \mathrm{C}_{6}-\mathrm{H}\right), 8.20\left(\mathrm{dd}, 1 \mathrm{H}, \mathrm{J}=1.5,5.7 \mathrm{~Hz}, \mathrm{C}_{9}, \mathrm{H}\right)$, $8.24\left(\mathrm{~d}, 1 \mathrm{H}, \mathrm{J}=6.2 \mathrm{~Hz}, \mathrm{C}_{5}-\mathrm{H}\right), 8.47\left(\mathrm{dd}, 1 \mathrm{H}, \mathrm{J}=1.5,5.7 \mathrm{~Hz}, \mathrm{C}_{3}-\mathrm{H}\right), 9.87$ $\left(\mathrm{s}, 1 \mathrm{H}, \mathrm{C}_{1}-\mathrm{H}\right)$.

(Isoquinolin-2-ium-2-yl)(6-trifluoromethyl nicotinoyl)amide (5w): Yield 64\%; mp 195.2-195.6 ${ }^{\circ} \mathrm{C} ;{ }^{1} \mathrm{HNMR}\left(\mathrm{CDCl}_{3}\right) \delta$ (ppm): 7.03 (d, $\left.1 \mathrm{H}, \mathrm{J}=6 . .9 \mathrm{~Hz}, \mathrm{C}_{7}-\mathrm{H}\right), 7.14-7.18\left(\mathrm{~m}, 3 \mathrm{H}, \mathrm{C}_{5}, \mathrm{C}_{6}, \mathrm{C}_{8}-\mathrm{H}\right), 7.75(\mathrm{~d}, 1 \mathrm{H}$, $\left.\mathrm{C}_{4^{\prime}}-\mathrm{H}\right), 8.10\left(\mathrm{~d}, 1 \mathrm{H}, \mathrm{J}=8.1 \mathrm{~Hz}, \mathrm{C}_{4}-\mathrm{H}\right), 8.31\left(\mathrm{~d}, 1 \mathrm{H}, \mathrm{J}=8.4 \mathrm{~Hz}, \mathrm{C}_{5^{-}}-\mathrm{H}\right), 8.44$ (dd, $\left.1 \mathrm{H}, \mathrm{J}=1.5,5.7 \mathrm{~Hz}, \mathrm{C}_{3}-\mathrm{H}\right), 9.03\left(\mathrm{~s}, 1 \mathrm{H}, \mathrm{C}_{2}-\mathrm{H}\right), 9.89$ (s, $\left.1 \mathrm{H}, \mathrm{C}_{1}-\mathrm{H}\right)$.

(2,6-Dichloro-5-fluoronicotinoyl)(isoquinolin-2-ium-2-yl)amide (5x): Yield 60\%; mp 198.5-200.5 ${ }^{\circ} \mathrm{C} ;{ }^{1} \mathrm{HNMR}\left(\mathrm{CDCl}_{3}\right) \delta(\mathrm{ppm}): 7.03(\mathrm{~d}$, $\left.1 \mathrm{H}, \mathrm{J}=6.9 \mathrm{~Hz}, \mathrm{C}_{7}-\mathrm{H}\right), 7.16-7.20\left(\mathrm{~m}, 3 \mathrm{H}, \mathrm{C}_{5}, \mathrm{C}_{6}, \mathrm{C}_{8}-\mathrm{H}\right), 7.92(\mathrm{~d}, 1 \mathrm{H}, \mathrm{J}=6.0$ $\left.\mathrm{Hz}, \mathrm{C}_{4}-\mathrm{H}\right), 8.15\left(\mathrm{~d}, 1 \mathrm{H}, \mathrm{J}=8.1 \mathrm{~Hz}, \mathrm{C}_{4}-\mathrm{H}\right), 8.48(\mathrm{dd}, 1 \mathrm{H}, \mathrm{J}=1.5,5.7 \mathrm{~Hz}$, $\left.\mathrm{C}_{3}-\mathrm{H}\right), 9.91\left(\mathrm{~s}, 1 \mathrm{H}, \mathrm{C}_{1}-\mathrm{H}\right)$.

General procedure for reduction (6a-x): A solution of Ylide (5 $\mathrm{mmol}$ ) in $20 \mathrm{ml}$ of absolute ethanol was added drop-wise to a solution of sodium borohydride ( $50 \mathrm{mmmol}$ ) in $25 \mathrm{ml}$ of absolute ethanol precooled to $0{ }^{\circ} \mathrm{C}$. The reaction was allowed to proceed for $5-7 \mathrm{~h}$ at $0^{\circ} \mathrm{C}$ with stirring. Water $(35 \mathrm{ml})$ was added, and allowed to warm up to room temperature. Extraction with dichloromethane $(3 \times 50 \mathrm{ml})$, drying over anhydrous sodium sulfate, and removal of the solvent in vacuo gave the crude product, which was purified on Combiflash using ethyl acetate: dichloromethane $(2: 3 \mathrm{v} / \mathrm{v})$ as an eluent to afford a pure compounds 6a-w in fair to good yields.

$N$-(1,2,3,4-tetrahydroisoquinolin-2-yl)benzamide $\quad(6 a)$ : Yield 60\%; mp 197.4-198.7 ${ }^{\circ} \mathrm{C}$; ${ }^{1} \mathrm{HNMR}\left(\mathrm{CDCl}_{3}\right) \delta$ (ppm): $3.06(\mathrm{t}, 2 \mathrm{H}, J=5.7$ $\left.\mathrm{Hz}, \mathrm{C}_{4}-\mathrm{H}\right), 3.34\left(\mathrm{t}, 2 \mathrm{H}, J=6.0 \mathrm{~Hz}, \mathrm{C}_{3}-\mathrm{H}\right), 4.21\left(\mathrm{~s}, 2 \mathrm{H}, \mathrm{C}_{1}-\mathrm{H}\right), 7.01(\mathrm{~d}, 1 \mathrm{H}$, $\left.J=3.0 \mathrm{~Hz}, \mathrm{C}_{8}-\mathrm{H}\right), 7.08\left(\mathrm{~s}, 1 \mathrm{H},-\mathrm{NH}_{2}, \mathrm{D}_{2} \mathrm{O}\right.$ exchange $), 7.14-7.18(\mathrm{~m}, 3 \mathrm{H}$, $\left.\mathrm{C}_{3}, \mathrm{C}_{4}, \mathrm{C}_{5^{\prime}}, \mathrm{H}\right), 8.31\left(\mathrm{~d}, 1 \mathrm{H}, J=6.9 \mathrm{~Hz}, \mathrm{C}_{4}-\mathrm{H}\right), 7.39-7.50\left(\mathrm{~m}, 3 \mathrm{H}, \mathrm{C}_{6}, \mathrm{C}_{7}\right.$, $\left.\mathrm{C}_{9}-\mathrm{H}\right), 7.75\left(\mathrm{~d}, 2 \mathrm{H}, J=7.5 \mathrm{~Hz}, \mathrm{C}_{2}, \mathrm{C}_{6}-\mathrm{H}\right)$. Anal. Calcd. for $\mathrm{C}_{16} \mathrm{H}_{16} \mathrm{~N}_{2} \mathrm{O}: \mathrm{C}$, 76.16; H, 6.39; N, 11.10. Found: C, 75.97; H, 6.28; N, 11.05 .

4-Methyl-N-(1,2,3,4-tetrahydroisoquinolin-2-yl)benzamide (6b):Yield 65\%; mp 195.9-196.4 ${ }^{\circ} \mathrm{C}$; ${ }^{1} \mathrm{HNMR}\left(\mathrm{CDCl}_{3}\right) \delta$ (ppm): 2.39 (s, $3 \mathrm{H}, \mathrm{CH}_{3}$ group), $3.06\left(\mathrm{t}, 2 \mathrm{H}, J=5.7 \mathrm{~Hz}, \mathrm{C}_{4}-\mathrm{H}\right), 3.35(\mathrm{t}, 2 \mathrm{H}, \mathrm{J}=6.0 \mathrm{~Hz}$, $\left.\mathrm{C}_{3}-\mathrm{H}\right), 4.2\left(\mathrm{~s}, 2 \mathrm{H}, \mathrm{C}_{1}-\mathrm{H}\right), 6.98\left(\mathrm{~d}, 2 \mathrm{H}, \mathrm{J}=6.0 \mathrm{~Hz}, \mathrm{C}_{3}, \mathrm{C}_{5}-\mathrm{H}\right), 7.01(\mathrm{~d}$, $\left.1 \mathrm{H}, J=3.0 \mathrm{~Hz}, \mathrm{C}_{8}-\mathrm{H}\right), 7.04\left(\mathrm{~s}, 1 \mathrm{H},-\mathrm{NH}_{2}, \mathrm{D}_{2} \mathrm{O}\right.$ exchange), 7.14-7.20 (m, 
Citation: Gangapuram M, Eyunni S, Redda KK (2014) Synthesis and Pharmacological Evolution of Tetrahydroisoquinolines as Anti Breast Cancer Agents. J Cancer Sci Ther 6: 161-169. doi:10.4172/1948-5956.1000266

$\left.3 \mathrm{H}, \mathrm{C}_{6}, \mathrm{C}_{7}, \mathrm{C}_{9}-\mathrm{H}\right), 7.75\left(\mathrm{~d}, 2 \mathrm{H}, J=8.4 \mathrm{~Hz}, \mathrm{C}_{2^{\prime}}, \mathrm{C}_{6}-\mathrm{H}\right)$. Anal. Calcd. for $\mathrm{C}_{17} \mathrm{H}_{18} \mathrm{~N}_{2} \mathrm{O}: \mathrm{C}, 76.66 ; \mathrm{H}, 6.81 ; \mathrm{N}, 10.52$. Found: $\mathrm{C}, 76.47 ; \mathrm{H}, 6.69 ; \mathrm{N}$, 10.46 .

4-Methoxy-N-(1,2,3,4-tetrahydroisoquinolin-2-yl)benzamide (6c): Yield 60\%; mp 197.3-199.1 ${ }^{\circ} \mathrm{C} ;{ }^{1} \mathrm{HNMR}\left(\mathrm{CDCl}_{3}\right) \delta(\mathrm{ppm}): 3.05(\mathrm{t}$, $\left.2 \mathrm{H}, J=5.7 \mathrm{~Hz}, \mathrm{C}_{4}-\mathrm{H}\right), 3.33\left(\mathrm{t}, 2 \mathrm{H}, J=6.0 \mathrm{~Hz}, \mathrm{C}_{3}-\mathrm{H}\right), 3.84\left(\mathrm{~s}, 3 \mathrm{H}, \mathrm{OCH}_{3}\right.$ group), $4.21\left(\mathrm{~s}, 2 \mathrm{H} \mathrm{C}_{1}-\mathrm{H}\right), 6.90\left(\mathrm{~d}, 2 \mathrm{H}, J=9.0 \mathrm{~Hz}, \mathrm{C}_{3^{\prime}}, \mathrm{C}_{5^{\prime}}-\mathrm{H}\right), 7.01(\mathrm{~d}$, $\left.1 \mathrm{H}, J=3.0 \mathrm{~Hz}, \mathrm{C}_{8}-\mathrm{H}\right), 7.04\left(\mathrm{~s}, 1 \mathrm{H},-\mathrm{NH}_{2}, \mathrm{D}_{2} \mathrm{O}\right.$ exchange), 7.14-7.28 (m, $\left.3 \mathrm{H}, \mathrm{C}_{6}, \mathrm{C}_{7}, \mathrm{C}_{9}-\mathrm{H}\right), 7.72\left(\mathrm{~d}, 2 \mathrm{H} \mathrm{J}=8.1 \mathrm{~Hz}, \mathrm{C}_{2}, \mathrm{C}_{6}-\mathrm{H}\right)$. Anal. Calcd. for $\mathrm{C}_{17} \mathrm{H}_{18} \mathrm{~N}_{2} \mathrm{O}_{2}: \mathrm{C}, 72.32 ; \mathrm{H}, 6.43 ; \mathrm{N}, 9.92$. Found: C, 72.03; H, 6.25; N, 9.81.

4-Ethyl-N-(1,2,3,4-tetrahydroisoquinolin-2-yl)benzamide (6d): Yield 58.8\%; mp 170.1-172.0 ${ }^{\circ} \mathrm{C}$; ${ }^{1} \mathrm{HNMR}\left(\mathrm{CDCl}_{3}\right) \delta(\mathrm{ppm}): 1.24(\mathrm{t}, 3 \mathrm{H}$, $\left.\mathrm{J}=7.5 \mathrm{~Hz},-\mathrm{CH}_{2}-\mathrm{CH}_{3}\right), 2.64-2.72\left(\mathrm{q}, 2 \mathrm{H}, \mathrm{J}=7.8 \mathrm{~Hz},-\mathrm{CH}_{2-} \mathrm{CH}_{3}\right), 3.06(\mathrm{t}$, $\left.2 \mathrm{H}, \mathrm{J}=6.0 \mathrm{~Hz}, \mathrm{C}_{4}-\mathrm{H}\right), 3.31\left(\mathrm{t}, 2 \mathrm{H}, \mathrm{J}=5.7 \mathrm{~Hz}, \mathrm{C}_{3}-\mathrm{H}\right), 4.19\left(\mathrm{~s}, 2 \mathrm{H}, \mathrm{C}_{1}-\mathrm{H}\right)$, $7.01\left(\mathrm{~d}, 1 \mathrm{H}, \mathrm{J}=2.4 \mathrm{~Hz}, \mathrm{C}_{7}-\mathrm{H}\right), 7.03\left(\mathrm{~s}, 1 \mathrm{H},-\mathrm{NH}, \mathrm{D}_{2} \mathrm{O}\right.$ exchange), 7.13$7.18\left(\mathrm{~m}, 3 \mathrm{H}, \mathrm{C}_{5}, \mathrm{C}_{6}, \mathrm{C}_{8}-\mathrm{H}\right), 7.24\left(\mathrm{~d}, 2 \mathrm{H}, \mathrm{J}=8.1 \mathrm{~Hz}, \mathrm{C}_{3}, \mathrm{C}_{5}, \mathrm{H}\right), 7.67(\mathrm{~d}, 2 \mathrm{H}$, $\left.\mathrm{J}=8.1 \mathrm{~Hz}, \mathrm{C}_{2}, \mathrm{C}_{6}-\mathrm{H}\right)$. Anal. Calcd. for $\mathrm{C}_{18} \mathrm{H}_{20} \mathrm{~N}_{20} \mathrm{O}: \mathrm{C}, 77.11 ; \mathrm{H}, 7.19 ; \mathrm{N}$, 9.99. Found: C, 76.88; H, 7.13; N, 9.94.

4-Propyl-N-(1,2,3,4-tetrahydroisoquinolin-2-yl)benzamide (6e): Yield 64.3\%; mp 160.2-161.3 ${ }^{\circ} \mathrm{C}$; ${ }^{1} \mathrm{HNMR}\left(\mathrm{CDCl}_{3}\right) \delta(\mathrm{ppm}): 0.92(\mathrm{t}, 3 \mathrm{H}$, $\left.\mathrm{J}=7.2 \mathrm{~Hz},-\mathrm{CH}_{2}-\mathrm{CH}_{2}-\mathrm{CH}_{3}\right), 1.59-1.66\left(\mathrm{~m}, 2 \mathrm{H},-\mathrm{CH}_{2}-\mathrm{CH}_{2}-\mathrm{CH}_{3}\right), 2.61(\mathrm{t}$, $\left.2 \mathrm{H}, \mathrm{J}=8.1 \mathrm{~Hz},-\mathrm{CH}_{2}-\mathrm{CH}_{2}-\mathrm{CH}_{3}\right), 3.15\left(\mathrm{t}, 2 \mathrm{H}, \mathrm{J}=5.7 \mathrm{~Hz}, \mathrm{C}_{4}-\mathrm{H}\right), 3.66(\mathrm{t}, 2 \mathrm{H}$, $\left.\mathrm{J}=5.7 \mathrm{~Hz}, \mathrm{C}_{3}-\mathrm{H}\right), 4.54\left(\mathrm{~s}, 2 \mathrm{H}, \mathrm{C}_{1}-\mathrm{H}\right), 6.99\left(\mathrm{~d}, 1 \mathrm{H}, \mathrm{J}=6.6 \mathrm{~Hz}, \mathrm{C}_{7}-\mathrm{H}\right), 7.18$ (s, $1 \mathrm{H},-\mathrm{NH}, \mathrm{D}_{2} \mathrm{O}$ exchange), 7.13-7.18 (m, 3H, $\left.\mathrm{C}_{5}, \mathrm{C}_{6}, \mathrm{C}_{8}-\mathrm{H}\right), 7.21(\mathrm{~d}$, $\left.2 \mathrm{H}, \mathrm{J}=8.4 \mathrm{~Hz}, \mathrm{C}_{3}, \mathrm{C}_{5}, \mathrm{H}\right), 7.75\left(\mathrm{~d}, 2 \mathrm{H}, \mathrm{J}=8.4 \mathrm{~Hz}, \mathrm{C}_{2}, \mathrm{C}_{6},-\mathrm{H}\right)$. Anal. Calcd. for $\mathrm{C}_{19} \mathrm{H}_{22} \mathrm{~N}_{2} \mathrm{O}$ : C, 63.55; H, 6.00; N, 9.26. Found: C, 63.28; H, 5.85; N, 9.32 .

4-Butyl-N-(1,2,3,4-tetrahydroisoquinolin-2-yl)benzamide (6f): Yield 68.2\%; mp 213.3-214.8 ${ }^{\circ} \mathrm{C} ;{ }^{1} \mathrm{HNMR}\left(\mathrm{CDCl}_{3}\right) \delta(\mathrm{ppm}): 0.93(\mathrm{t}, 3 \mathrm{H}$, $\left.\mathrm{J}=7.5 \mathrm{~Hz},-\mathrm{CH}_{2}-\mathrm{CH}_{2}-\mathrm{CH}_{2}-\mathrm{CH}_{3}\right), 1.36\left(\mathrm{q}, 2 \mathrm{H}, \mathrm{J}=7.2 \mathrm{~Hz},-\mathrm{CH}_{2}-\mathrm{CH}_{2}-\mathrm{CH}_{2}-\right.$ $\left.\mathrm{CH}_{3}\right), 1.57-1.67\left(\mathrm{~m}, 2 \mathrm{H},-\mathrm{CH}_{2}-\mathrm{CH}_{2}-\mathrm{CH}_{2}-\mathrm{CH}_{3}\right), 2.69(\mathrm{t}, 2 \mathrm{H}, \mathrm{J}=7.5, \mathrm{~Hz}$, $\left.-\mathrm{CH}_{2}-\mathrm{CH}_{2}-\mathrm{CH}_{2}-\mathrm{CH}_{3}\right) 3.19\left(\mathrm{t}, 2 \mathrm{H}, \mathrm{J}=5.8 \mathrm{~Hz}, \mathrm{C}_{4}-\mathrm{H}\right), 3.70(\mathrm{t}, 2 \mathrm{H}, \mathrm{J}=6.0$ $\left.\mathrm{Hz}, \mathrm{C}_{3}-\mathrm{H}\right), 4.58\left(\mathrm{~s}, 2 \mathrm{H}, \mathrm{C}_{1}-\mathrm{H}\right), 7.01\left(\mathrm{~d}, 1 \mathrm{H}, \mathrm{J}=6.2 \mathrm{~Hz}, \mathrm{C}_{7}-\mathrm{H}\right), 7.19(\mathrm{~s}$, $1 \mathrm{H},-\mathrm{NH}, \mathrm{D}_{2} \mathrm{O}$ exchange), 7.15-7.21 (m, 3H, $\left.\mathrm{C}_{5}, \mathrm{C}_{6}, \mathrm{C}_{8}-\mathrm{H}\right), 7.26(\mathrm{~d}, 2 \mathrm{H}$, $\left.\mathrm{J}=8.4 \mathrm{~Hz}, \mathrm{C}_{3}, \mathrm{C}_{5},-\mathrm{H}\right), 7.81\left(\mathrm{~d}, 2 \mathrm{H}, \mathrm{J}=8.5 \mathrm{~Hz}, \mathrm{C}_{2}, \mathrm{C}_{6}-\mathrm{H}\right)$. Anal. Calcd. for $\mathrm{C}_{20} \mathrm{H}_{24} \mathrm{~N}_{2} \mathrm{O}: \mathrm{C}, 77.89 ; \mathrm{H}, 7.94 ; \mathrm{N}, 9.08$. Found: C, 77.80; H, 7.89; N, 8.99.

4-(tert-Butyl)-N-(1,2,3,4-tetrahydroisoquinolin-2-yl)benzamide (6g): Yield 68.2\%; mp 213.3-214.8 ${ }^{\circ} \mathrm{C} ;{ }^{1} \mathrm{HNMR}\left(\mathrm{CDCl}_{3}\right) \delta$ (ppm): 1.32 (s, 9H, $t$-butyl group), $3.06\left(\mathrm{t}, 2 \mathrm{H}, \mathrm{J}=5.4 \mathrm{~Hz}, \mathrm{C}_{4}-\mathrm{H}\right), 3.31(\mathrm{t}, 2 \mathrm{H}, \mathrm{J}=6.0$ $\left.\mathrm{Hz}, \mathrm{C}_{3}-\mathrm{H}\right), 4.20\left(\mathrm{~s}, 2 \mathrm{H}, \mathrm{C}_{1}-\mathrm{H}\right), 7.02\left(\mathrm{dd}, 1 \mathrm{H}, \mathrm{J}=2.7,5.4 \mathrm{~Hz}, \mathrm{C}_{7}-\mathrm{H}\right), 7.09$ (s, $1 \mathrm{H},-\mathrm{NH}, \mathrm{D}_{2} \mathrm{O}$ exchange), 7.11-1.18 (m, 3H, $\left.\mathrm{C}_{5}, \mathrm{C}_{6}, \mathrm{C}_{8}-\mathrm{H}\right), 7.44(\mathrm{~d}$, $\left.2 \mathrm{H}, \mathrm{J}=8.7 \mathrm{~Hz}, \mathrm{C}_{3}, \mathrm{C}_{5}, \mathrm{H}\right), 7.68\left(\mathrm{~d}, 2 \mathrm{H}, \mathrm{J}=8.4 \mathrm{~Hz}, \mathrm{C}_{2}, \mathrm{C}_{6},-\mathrm{H}\right)$. Anal. Calcd. for $\mathrm{C}_{20} \mathrm{H}_{24} \mathrm{~N}_{2} \mathrm{O} 0.05 \mathrm{EtOAc}$ C, $76.50 ; \mathrm{H}, 7.68 ; \mathrm{N}, 8.81$. Found: $\mathrm{C}, 76.79$; $\mathrm{H}, 7.73 ; \mathrm{N}, 9.95$.

4-Fluoro-N-(1,2,3,4-tetrahydroisoquinolin-2-yl)benzamide (6h): Yield 58.6\%; mp 200.4-201.6 ${ }^{\circ} \mathrm{C}$; ${ }^{1} \mathrm{HNMR}\left(\mathrm{CDCl}_{3}\right) \delta(\mathrm{ppm}): 3.07(\mathrm{t}, 2 \mathrm{H}$, $\left.\mathrm{J}=6.0 \mathrm{~Hz}, \mathrm{C}_{4}-\mathrm{H}\right), 3.32\left(\mathrm{t}, 2 \mathrm{H}, \mathrm{J}=6.0 \mathrm{~Hz}, \mathrm{C}_{3}-\mathrm{H}\right), 4.19\left(\mathrm{~s}, 2 \mathrm{H}, \mathrm{C}_{1}-\mathrm{H}\right), 7.02$ (d, $\left.1 \mathrm{H}, \mathrm{J}=6.3,2.1 \mathrm{~Hz}, \mathrm{C}_{7}-\mathrm{H}\right), 7.08$ (s, $1 \mathrm{H},-\mathrm{NH}, \mathrm{D}_{2} \mathrm{O}$ exchange), 7.11-7.18 $\left(\mathrm{m}, 3 \mathrm{H}, \mathrm{C}_{5}, \mathrm{C}_{6} \mathrm{C}_{8}-\mathrm{H}\right), 7.19\left(\mathrm{~d}, 2 \mathrm{H}, \mathrm{J}=6.3 \mathrm{~Hz}, \mathrm{C}_{3}, \mathrm{C}_{5},-\mathrm{H}\right), 7.76(\mathrm{~d}, 2 \mathrm{H}, \mathrm{J}=7.2$ $\left.\mathrm{Hz}, \mathrm{C}_{2}, \mathrm{C}_{6}-\mathrm{H}\right)$. Anal. Calcd. for $\mathrm{C}_{16} \mathrm{H}_{15} \mathrm{FN}_{2} \mathrm{O}: \mathrm{C}, 71.10 ; \mathrm{H}, 5.59 ; \mathrm{N}, 10.36$. Found: C, 70.86; H, 5.61; N, 10.46 .

4-Chloro-N-(1,2,3,4-tetrahydroisoquinolin-2-yl)benzamide (6i): Yield 70\%; mp 165.1-167.3 ${ }^{\circ} \mathrm{C}$; ${ }^{1} \mathrm{HNMR}\left(\mathrm{CDCl}_{3}\right) \delta$ (ppm): $3.07(\mathrm{t}, 2 \mathrm{H}$, $\left.J=5.7 \mathrm{~Hz}, \mathrm{C}_{4}-\mathrm{H}\right), 3.32\left(\mathrm{t}, 2 \mathrm{H}, J=6.0 \mathrm{~Hz}, \mathrm{C}_{3}-\mathrm{H}\right), 4.19\left(\mathrm{~s}, 2 \mathrm{H}, \mathrm{C}_{1}-\mathrm{H}\right), 7.01$ (d, $\left.2 \mathrm{H}, J=9.0 \mathrm{~Hz}, \mathrm{C}_{3,}, \mathrm{C}_{5}, \mathrm{H}\right), 7.05$ (d, $\left.1 \mathrm{H}, J=3.0 \mathrm{~Hz}, \mathrm{C}_{8}-\mathrm{H}\right), 7.08$ (s, 1H., $-\mathrm{NH}_{2}, \mathrm{D}_{2} \mathrm{O}$ exchange), 7.14-7.18 (m, 3H, C, $\left.\mathrm{C}_{7}, \mathrm{C}_{9}-\mathrm{H}\right), 7.70(\mathrm{~d}, 2 \mathrm{H}$,
$\left.J=8.1 \mathrm{~Hz}, \mathrm{C}_{3^{\prime}}, \mathrm{C}_{6}-\mathrm{H}\right)$. Anal. Calcd. for $\mathrm{C}_{16} \mathrm{H}_{15} \mathrm{ClN}_{2} \mathrm{O}: \mathrm{C}, 67.02 ; \mathrm{H}, 5.27$; N, 9.77. Found: C, 67.12; H, 5.19; N, 9.69.

4-Bromo-N-(1,2,3,4-tetrahydroisoquinolin-2-yl)benzamide (6j): Yield 55.6\%; mp 199.8-200.9 ${ }^{\circ} \mathrm{C} ;{ }^{1} \mathrm{HNMR}\left(\mathrm{CDCl}_{3}\right) \delta(\mathrm{ppm}): 3.07(\mathrm{t}, 2 \mathrm{H}$, $\left.\mathrm{J}=5.7 \mathrm{~Hz}, \mathrm{C}_{4}-\mathrm{H}\right), 3.32\left(\mathrm{t}, 2 \mathrm{H}, \mathrm{J}=6.0 \mathrm{~Hz}, \mathrm{C}_{3}-\mathrm{H}\right), 4.19\left(\mathrm{~s}, 2 \mathrm{H}, \mathrm{C}_{1}-\mathrm{H}\right), 7.02$ (d, $\left.1 \mathrm{H}, J=2.4 \mathrm{~Hz}, \mathrm{C}_{7}-\mathrm{H}\right), 7.04\left(\mathrm{~s}, 1 \mathrm{H},-\mathrm{NH}, \mathrm{D}_{2} \mathrm{O}\right.$ exchange), 7.14-7.19 (m, $\left.3 \mathrm{H}, \mathrm{C}_{5}, \mathrm{C}_{6} \mathrm{C}_{8}-\mathrm{H}\right), 7.26\left(\mathrm{~d}, 2 \mathrm{H}, \mathrm{J}=8.2 \mathrm{~Hz}, \mathrm{C}_{3}, \mathrm{C}_{5}, \mathrm{H}\right), 7.69$ (d, 2H, J=8.1 $\left.\mathrm{Hz}, \mathrm{C}_{2}, \mathrm{C}_{6}-\mathrm{H}\right)$. Anal. Calcd. for $\mathrm{C}_{16} \mathrm{H}_{15} \mathrm{BrN}_{2} \mathrm{O} 0.2$ EtOAc: C, 55.01; $\mathrm{H}$, $4.31 ; \mathrm{N}, 7.83$. Found: C, 55.09; H, 4.33; N, 8.03.

$\mathrm{N}$-(1,2,3,4-tetrahydroisoquinolin-2-yl)-4-(trifluoromethyl) benzamide(6k): Yield 60.4\%; mp 195.9-196.9 ${ }^{\circ} \mathrm{C}$; ${ }^{1} \mathrm{HNMR}\left(\mathrm{CDCl}_{3}\right)$ $\delta(\mathrm{ppm}): 3.07\left(\mathrm{t}, 2 \mathrm{H}, \mathrm{J}=6.0 \mathrm{~Hz}, \mathrm{C}_{4}-\mathrm{H}\right), 3.35\left(\mathrm{t}, 2 \mathrm{H}, \mathrm{J}=5.7 \mathrm{~Hz}, \mathrm{C}_{3}-\mathrm{H}\right)$, $4.21\left(\mathrm{~s}, 1 \mathrm{H}, \mathrm{C}_{1}-\mathrm{H}\right), 7.03\left(\mathrm{~d}, 1 \mathrm{H}, \mathrm{J}=5.7 \mathrm{~Hz}, \mathrm{C}_{7}-\mathrm{H}\right), 7.14\left(\mathrm{~s}, 1 \mathrm{H},-\mathrm{NH}, \mathrm{D}_{2} \mathrm{O}\right.$ exchange), 7.16-7.19 (m, 3H, $\left.\mathrm{C}_{5}, \mathrm{C}_{6}, \mathrm{C}_{8}-\mathrm{H}\right), 7.69\left(\mathrm{~d}, 2 \mathrm{H}, \mathrm{J}=8.1 \mathrm{~Hz}, \mathrm{C}_{3}\right.$, $\left.\mathrm{C}_{5}, \mathrm{H}\right), 7.86\left(\mathrm{~d}, 2 \mathrm{H}, \mathrm{J}=8.1 \mathrm{~Hz}, \mathrm{C}_{2}, \mathrm{C}_{6}-\mathrm{H}\right)$. Anal. Calcd. for $\mathrm{C}_{17} \mathrm{H}_{15} \mathrm{~F}_{3} \mathrm{~N}_{2} \mathrm{O}$ : C, $63.75 ; \mathrm{H}, 4.72 ; \mathrm{N}, 8.75$. Found: C, 63.64; H, 4.62; N, 8.71.

$N-(1,2,3,4-t e t r a h y d r o i s o q u i n o l i n-2-y l)-3,4,5-$ trimethoxybenzamide (6l): Yield 59.5\%; mp 216.3-217. ${ }^{\circ} \mathrm{C}$; ${ }^{1} \mathrm{HNMR}$ $\left(\mathrm{CDCl}_{3}\right) \delta(\mathrm{ppm}): 3.17\left(\mathrm{t}, 2 \mathrm{H}, \mathrm{J}=5.7 \mathrm{~Hz}, \mathrm{C}_{4}-\mathrm{H}\right), 3.34(\mathrm{t}, 2 \mathrm{H}, \mathrm{J}=6.0 \mathrm{~Hz}$, $\mathrm{C}_{3}-\mathrm{H}$ ), 3.86 (s, $3 \mathrm{H}, p-\mathrm{OCH}_{3}$ group), 3.90 (s, $6 \mathrm{H}, m-\mathrm{OCH}_{3}$ group), 4.22 (s, 2H, C $-\mathrm{H}), 6.96\left(\mathrm{~s}, 2 \mathrm{H}, \mathrm{C}_{2}, \mathrm{C}_{6}-\mathrm{H}\right), 7.03\left(\mathrm{~d}, 1 \mathrm{H}, \mathrm{J}=3.9 \mathrm{~Hz}, \mathrm{C}_{7}-\mathrm{H}\right), 7.14$ (s, $1 \mathrm{H},-\mathrm{NH}, \mathrm{D}_{2} \mathrm{O}$ exchange), 7.15-7.19 (m, 3H, $\left.\mathrm{C}_{5}, \mathrm{C}_{6}, \mathrm{C}_{8}-\mathrm{H}\right)$. Anal. Calcd. for $\mathrm{C}_{19} \mathrm{H}_{22} \mathrm{~N}_{2} \mathrm{O}_{4}$ : C, 66.65; H, 6.48; N, 8.18. Found: C, 66.91; $\mathrm{H}$, $6.42 ; \mathrm{N}, 8.44$.

4-Nitro-N-(1,2,3,4-tetrahydroisoquinolin-2-yl)benzamide (6m): Yield 48\%; mp 184.0-185.9 ${ }^{\circ} \mathrm{C}$; ${ }^{1} \mathrm{HNMR}\left(\mathrm{CDCl}_{3}\right) \delta$ (ppm): $3.13(\mathrm{t}, 2 \mathrm{H}$, $\left.\mathrm{J}=5.7 \mathrm{~Hz}, \mathrm{C}_{4}-\mathrm{H}\right), 3.49\left(\mathrm{t}, 2 \mathrm{H}, \mathrm{J}=5.7 \mathrm{~Hz}, \mathrm{C}_{3}-\mathrm{H}\right), 4.36\left(\mathrm{~s}, 2 \mathrm{H}, \mathrm{C}_{1}-\mathrm{H}\right), 7.04$ (d, $1 \mathrm{H}, \mathrm{J}=6.9 \mathrm{~Hz}, \mathrm{C}_{7}-\mathrm{H}$ ), 7.15 (s, $1 \mathrm{H},-\mathrm{NH}, \mathrm{D}_{2} \mathrm{O}$ exchange), 7.18-7.22 $\left(\mathrm{m}, 3 \mathrm{H}, \mathrm{C}_{5}, \mathrm{C}_{6}, \mathrm{C}_{8}-\mathrm{H}\right), 8.01\left(\mathrm{~d}, 2 \mathrm{H}, \mathrm{J}=8.7 \mathrm{~Hz}, \mathrm{C}_{3}, \mathrm{C}_{5},-\mathrm{H}\right), 8.28(\mathrm{~d}, 2 \mathrm{H}, \mathrm{J}=9.0$ $\left.\mathrm{Hz}, \mathrm{C}_{2}, \mathrm{C}_{6}-\mathrm{H}\right)$. Anal. Calcd. for $\mathrm{C}_{16} \mathrm{H}_{15} \mathrm{~N}_{3} \mathrm{O}_{3}: \mathrm{C}, 64.64 ; \mathrm{H}, 5.09 ; \mathrm{N}, 14.13$. Found: C, 64.58; H, 5.04; N, 14.20.

$N$-(1,2,3,4-tetrahydroisoquinolin-2-yl)benzenesulfonamide (6n): Yield 56.9\%; mp 132.5-133.3 ${ }^{\circ} \mathrm{C} ;{ }^{1} \mathrm{HNMR}\left(\mathrm{CDCl}_{3}\right) \delta(\mathrm{ppm}): 2.84(\mathrm{t}, 2 \mathrm{H}$, $\left.\mathrm{J}=6.3 \mathrm{~Hz}, \mathrm{C}_{4}-\mathrm{H}\right), 2.93\left(\mathrm{t}, 2 \mathrm{H}, \mathrm{J}=5.1 \mathrm{~Hz}, \mathrm{C}_{3}-\mathrm{H}\right), 3.83\left(\mathrm{~s}, 2 \mathrm{H}, \mathrm{C}_{1}-\mathrm{H}\right), 5.55$ (s, $1 \mathrm{H},-\mathrm{NH}, \mathrm{D}_{2} \mathrm{O}$ exchange), $6.88\left(\mathrm{dd}, 1 \mathrm{H}, \mathrm{J}=2.1,6.6 \mathrm{~Hz}, \mathrm{C}_{6}-\mathrm{H}\right), 7.05-$ $7.16\left(\mathrm{~m}, 3 \mathrm{H}, \mathrm{C}_{3}, \mathrm{C}_{4}, \mathrm{C}_{5}, \mathrm{H}\right), 7.47 .55\left(\mathrm{~m}, 2 \mathrm{H}, \mathrm{C}_{5}, \mathrm{C}_{8}-\mathrm{H}\right), 7.61(\mathrm{ttt}, 1 \mathrm{H}$, $\left.\mathrm{J}=1.5,2.4,2.4 \mathrm{~Hz}, \mathrm{C}_{7}-\mathrm{H}\right), 7.99\left(\mathrm{dd}, 2 \mathrm{H}, \mathrm{J}=1.5,5.7 \mathrm{~Hz}, \mathrm{C}_{2}, \mathrm{C}_{6}-\mathrm{H}\right)$. Anal. Calcd. for $\mathrm{C}_{15} \mathrm{H}_{16} \mathrm{~N}_{2} \mathrm{O}_{2} \mathrm{~S}$ : C, 62.48; H, 5.59; N, 9.71. Found: C, 62.38; H, $5.39 ; \mathrm{N}, 9.69$.

4-Methyl-N-(1,2,3,4-tetrahydroisoquinolin-2-yl) benzenesulfonamide (6o): Yield 52.5\%; mp 135.2-135.7 ${ }^{\circ} \mathrm{C} ;{ }^{1} \mathrm{HNMR}$ $\left(\mathrm{CDCl}_{3}\right) \delta(\mathrm{ppm}): 2.44\left(\mathrm{~s}, 3 \mathrm{H}, \mathrm{CH}_{3}\right.$ group), $2.84\left(\mathrm{t}, 2 \mathrm{H}, J=6.3 \mathrm{~Hz}, \mathrm{C}_{4}-\right.$ $\mathrm{H}), 2.93\left(\mathrm{t}, 2 \mathrm{H}, J=5.4 \mathrm{~Hz}, \mathrm{C}_{3}-\mathrm{H}\right), 3.84\left(\mathrm{~s}, 2 \mathrm{H}, \mathrm{C}_{1}-\mathrm{H}\right), 5.52\left(\mathrm{~s}, 1 \mathrm{H},-\mathrm{NH}_{2}\right.$, $\mathrm{D}_{2} \mathrm{O}$ exchange), $6.89\left(\mathrm{dd}, 1 \mathrm{H}, J=1.8,6.9 \mathrm{~Hz}, \mathrm{C}_{7}-\mathrm{H}\right), 7.05-7.15(\mathrm{~m}, 3 \mathrm{H}$, $\left.\mathrm{C}_{5}, \mathrm{C}_{6}, \mathrm{C}_{8}-\mathrm{H}\right), 7.30\left(\mathrm{~d}, 2 \mathrm{H}, J=8.7 \mathrm{~Hz}, \mathrm{C}_{3}, \mathrm{C}_{5}, \mathrm{H}\right), 7.85(\mathrm{~d}, 2 \mathrm{H}, J=8.4 \mathrm{~Hz}$, $\left.\mathrm{C}_{2}, \mathrm{C}_{6}-\mathrm{H}\right)$. Anal. Calcd. for $\mathrm{C}_{16} \mathrm{H}_{18} \mathrm{~N}_{2} \mathrm{O}_{2} \mathrm{~S}$ : C, 63.75; H, 4.72; N, 8.75. Found: C, 63.64; H, 4.62; N, 8.71.

4-Methoxy-N-(1,2,3,4-tetrahydroisoquinolin-2-yl) benzenesulfonamide (6p): Yield 58\%; mp197.4-198.7 ${ }^{\circ} \mathrm{C}$; ${ }^{1} \mathrm{HNMR}$ $\left(\mathrm{CDCl}_{3}\right) \delta(\mathrm{ppm}): 3.06\left(\mathrm{t}, 2 \mathrm{H}, J=5.1 \mathrm{~Hz}, \mathrm{C}_{4}-\mathrm{H}\right), 3.34(\mathrm{t}, 2 \mathrm{H}, \mathrm{J}=7.5 \mathrm{~Hz}$, $\left.\mathrm{C}_{3}-\mathrm{H}\right), 3.84\left(\mathrm{~s}, 3 \mathrm{H}, \mathrm{OCH}_{3}\right.$ group), $4.21\left(\mathrm{~s}, 2 \mathrm{H}, \mathrm{C}_{1}-\mathrm{H}\right), 6.91(\mathrm{~d}, 2 \mathrm{H}, J=9.0$ $\left.\mathrm{Hz}, \mathrm{C}_{3^{\prime}}, \mathrm{C}_{5^{\prime}}-\mathrm{H}\right), 7.01\left(\mathrm{~d}, 1 \mathrm{H}, J=2.1 \mathrm{~Hz}, \mathrm{C}_{8}-\mathrm{H}\right), 7.04\left(\mathrm{~s}, 1 \mathrm{H},-\mathrm{NH}_{2}, \mathrm{D}_{2} \mathrm{O}\right.$ exchange), 7.2 1-7.18 (m, 3H, C, $\left.\mathrm{C}_{7}, \mathrm{C}_{9}-\mathrm{H}\right), 7.72(\mathrm{~d}, 2 \mathrm{H}, J=7.8 \mathrm{~Hz}$, $\left.\mathrm{C}_{2^{\prime}}, \mathrm{C}_{6^{\prime}}-\mathrm{H}\right)$. Anal. Calcd. for $\mathrm{C}_{16} \mathrm{H}_{18} \mathrm{~N}_{2} \mathrm{O}_{3} \mathrm{~S}: \mathrm{C}, 60.36 ; \mathrm{H}, 5.70 ; \mathrm{N}, 8.80$. Found: C, 59.97; H, 5.65; N, 8.68. 
4-Ethyl-N-(1,2,3,4-tetrahydroisoquinolin-2-yl) benzenesulfonamide (6q): Yield 49.5\%; mp 137.1-138.3 ${ }^{\circ} \mathrm{C} ;{ }^{1} \mathrm{HNMR}$ $\left(\mathrm{CDCl}_{3}\right) \delta(\mathrm{ppm}): 1.27(\mathrm{t}, 3 \mathrm{H}, \mathrm{J}=7.5 \mathrm{~Hz},-\mathrm{CH} 2-\mathrm{CH} 3), 2.66-2.75(\mathrm{q}, 2 \mathrm{H}$, $\mathrm{J}=7.5 \mathrm{~Hz},-\mathrm{CH} 2-\mathrm{CH} 3), 2.84\left(\mathrm{t}, 2 \mathrm{H}, J=5.7 \mathrm{~Hz}, \mathrm{C}_{4}-\mathrm{H}\right), 2.94(\mathrm{t}, 2 \mathrm{H}, J=5.1$ $\left.\mathrm{Hz}, \mathrm{C}_{3}-\mathrm{H}\right), 3.83\left(\mathrm{~s}, 2 \mathrm{H}, \mathrm{C}_{1}-\mathrm{H}\right), 5.54\left(\mathrm{~s}, 1 \mathrm{H},-\mathrm{NH}, \mathrm{D}_{2} \mathrm{O}\right.$ exchange), 6.89 $\left(\mathrm{dd}, 1 \mathrm{H}, J=1.8,6.6 \mathrm{~Hz}, \mathrm{C}_{7}-\mathrm{H}\right), 7.05-7.15\left(\mathrm{~m}, 3 \mathrm{H}, \mathrm{C}_{5}, \mathrm{C}_{6}, \mathrm{C}_{8}-\mathrm{H}\right), 7.32(\mathrm{~d}$, $\left.2 \mathrm{H}, J=6.0 \mathrm{~Hz}, \mathrm{C}_{3}, \mathrm{C}_{5},-\mathrm{H}\right), 7.88\left(\mathrm{~d}, 2 \mathrm{H}, J=6.3 \mathrm{~Hz}, \mathrm{C}_{2}, \mathrm{C}_{6},-\mathrm{H}\right)$. Anal. Calcd. for $\mathrm{C}_{17} \mathrm{H}_{20} \mathrm{~N}_{2} \mathrm{O}_{2} \mathrm{~S}$ : C, 64.53; H, 6.37; N, 8.85. Found: C, 64.42; H, 6.33; $\mathrm{N}, 8.81$.

4-Butyl-N-(1,2,3,4-tetrahydroisoquinolin-2-yl) benzenesulfonamide (6r): Yield 60\%; $\mathrm{mp}$ 197.3-199.1 ${ }^{\circ} \mathrm{C}$; ${ }^{1} \mathrm{HNMR}$ $\left(\mathrm{CDCl}_{3}\right) \delta(\mathrm{ppm}): 0.93\left(\mathrm{t}, 3 \mathrm{H}, \mathrm{J}=7.5 \mathrm{~Hz},-\mathrm{CH}_{2}-\mathrm{CH}_{2}-\mathrm{CH}_{2}-\mathrm{CH}_{3}\right), 1.36(\mathrm{q}$, $\left.2 \mathrm{H}, \mathrm{J}=7.2 \mathrm{~Hz},-\mathrm{CH}_{2}-\mathrm{CH}_{2}-\mathrm{CH}_{2}-\mathrm{CH}_{3}\right), 1.57-1.67\left(\mathrm{~m}, 2 \mathrm{H},-\mathrm{CH}_{2}-\mathrm{CH}_{2}-\mathrm{CH}_{2}-\right.$ $\left.\mathrm{CH}_{3}\right), 2.69\left(\mathrm{t}, 2 \mathrm{H}, \mathrm{J}=7.5, \mathrm{~Hz},-\mathrm{CH}_{2}-\mathrm{CH}_{2}-\mathrm{CH}_{2}-\mathrm{CH}_{3}\right) 2.84(\mathrm{t}, 2 \mathrm{H}, \mathrm{J}=5.7$ $\left.\mathrm{Hz}, \mathrm{C}_{4}-\mathrm{H}\right), 2.93\left(\mathrm{t}, 2 \mathrm{H}, \mathrm{J}=5.4 \mathrm{~Hz}, \mathrm{C}_{3}-\mathrm{H}\right), 3.82\left(\mathrm{~s}, 2 \mathrm{H}, \mathrm{C}_{1}-\mathrm{H}\right), 5.54(\mathrm{~s}, 1 \mathrm{H}$, -NH, $\mathrm{D}_{2} \mathrm{O}$ exchange), $6.88\left(\mathrm{dd}, 1 \mathrm{H}, J=2.1,6.3 \mathrm{~Hz}, \mathrm{C}_{7}-\mathrm{H}\right), 7.07-7.15(\mathrm{~m}$, $\left.3 \mathrm{H}, \mathrm{C}_{5}, \mathrm{C}_{6}, \mathrm{C}_{8}-\mathrm{H}\right), 7.32\left(\mathrm{~d}, 2 \mathrm{H}, J=6.0 \mathrm{~Hz}, \mathrm{C}_{3}, \mathrm{C}_{5}, \mathrm{H}\right), 7.87$ (d, 2H, J=6.3 $\mathrm{Hz}, \mathrm{C}_{2}, \mathrm{C}_{6}-\mathrm{H}$ ). Anal. Calcd. for $\mathrm{C}_{19} \mathrm{H}_{24} \mathrm{~N}_{2} \mathrm{OS}$ : C, 66.25; H, 7.02; N, 8.13. Found: C, 66.12; H, 7.02; N, 8.16.

$N$-(1,2,3,4-tetrahydroisoquinolin-2-yl)-furan-2-carboxamide (6s): Yield 68\%; mp 153.8-154.5; ${ }^{1} \mathrm{HNMR}\left(\mathrm{CDCl}_{3}\right) \delta(\mathrm{ppm}): 3.08(\mathrm{t}, 2 \mathrm{H}$, $\left.\mathrm{J}=6.0 \mathrm{~Hz}, \mathrm{C}_{4}-\mathrm{H}\right), 3.37\left(\mathrm{t}, 2 \mathrm{H}, \mathrm{J}=6.0 \mathrm{~Hz}, \mathrm{C}_{3}-\mathrm{H}\right), 4.24\left(\mathrm{~s}, 2 \mathrm{H}, \mathrm{C}_{1}-\mathrm{H}\right), 6.51$ $\left(\mathrm{d}, 2 \mathrm{H}, \mathrm{J}=1.5 \mathrm{~Hz}, \mathrm{C}_{4^{\prime}}-\mathrm{H}\right), 7.02\left(\mathrm{~d}, 1 \mathrm{H}, \mathrm{J}=6.3 \mathrm{~Hz}, \mathrm{C}_{7}-\mathrm{H}\right), 7.15(\mathrm{~s}, 1 \mathrm{H},-\mathrm{NH}$, $\mathrm{D}_{2} \mathrm{O}$ exchange), 7.17-7.20 (m, 3H, $\left.\mathrm{C}_{6}, \mathrm{C}_{7}, \mathrm{C}_{8}-\mathrm{H}\right), 7.43\left(\mathrm{~s}, 2 \mathrm{H}, \mathrm{C}_{3}, \mathrm{H}\right), 7.64$ $\left(\mathrm{S}, 1 \mathrm{H}, \mathrm{C}_{5}, \mathrm{H}\right)$. Anal. Calcd. for $\mathrm{C}_{14} \mathrm{H}_{14} \mathrm{~N}_{2} \mathrm{O}_{2}: \mathrm{C}, 69.41 ; \mathrm{H}, 5.82 ; \mathrm{N}, 11.56$. Found: C, 69.18; H, 5.76; N, 11.54 .

6-Chloro- $N-(1,2,3,4-t e t r a h y d r o i s o q u i n o l i n-2-y l) n i c o t i n a m i d e$ (6t): Yield 60\%; mp 197.3-199.1 ${ }^{\circ} \mathrm{C} ;{ }^{1} \mathrm{HNMR}\left(\mathrm{CDCl}_{3}\right) \delta(\mathrm{ppm}): 3.10(\mathrm{t}$, $\left.2 \mathrm{H}, \mathrm{J}=6.0 \mathrm{~Hz}, \mathrm{C}_{4}-\mathrm{H}\right), 3.43\left(\mathrm{t}, 2 \mathrm{H}, \mathrm{J}=6.0 \mathrm{~Hz}, \mathrm{C}_{3}-\mathrm{H}\right), 4.29\left(\mathrm{~s}, 2 \mathrm{H}, \mathrm{C}_{1}-\mathrm{H}\right)$, $7.02\left(\mathrm{~d}, 1 \mathrm{H}, \mathrm{J}=5.7 \mathrm{~Hz}, \mathrm{C}_{7}-\mathrm{H}\right), 7.14\left(\mathrm{~s}, 1 \mathrm{H},-\mathrm{NH}, \mathrm{D}_{2} \mathrm{O}\right.$ exchange), 7.16$7.22\left(\mathrm{~m}, 3 \mathrm{H}, \mathrm{C}_{5}, \mathrm{C}_{6}, \mathrm{C}_{8}-\mathrm{H}\right), 7.38\left(\mathrm{~d}, 1 \mathrm{H}, \mathrm{J}=8.1 \mathrm{~Hz}, \mathrm{C}_{5}, \mathrm{H}\right), 8.13(\mathrm{~d}, 1 \mathrm{H}$, $\left.\mathrm{J}=8.1 \mathrm{~Hz}, \mathrm{C}_{4}-\mathrm{H}\right), 8.73\left(\mathrm{~s}, 1 \mathrm{H}, \mathrm{C}_{2},-\mathrm{H}\right)$. Anal. Calcd. for $\mathrm{C}_{15} \mathrm{H}_{14} \mathrm{ClN}_{3} \mathrm{O}: \mathrm{C}$, 62.61; H, 4.90; N, 14.60. Found: C, 62.43; H, 4.92; N, 14.46.

(2-Chloro-6-methyl)-N-(1,2,3,4-tetrahydroisoquinolin-2-yl)isonicotinamide (6u): Yield 65\%; $\mathrm{mp} 188.5-190.2^{\circ} \mathrm{C} ;{ }^{1} \mathrm{HNMR}\left(\mathrm{CDCl}_{3}\right)$ $\delta(\mathrm{ppm}): 2.59\left(\mathrm{~s}, 3 \mathrm{H},-\mathrm{CH}_{3}\right), 3.12\left(\mathrm{t}, 2 \mathrm{H}, \mathrm{J}=5.7 \mathrm{~Hz}, \mathrm{C}_{4}-\mathrm{H}\right), 3.47(\mathrm{t}, 2 \mathrm{H}$, $\left.\mathrm{J}=6.3 \mathrm{~Hz}, \mathrm{C}_{3}-\mathrm{H}\right), 4.34$ (s, 2H, $\left.\mathrm{C}_{1}-\mathrm{H}\right), 7.03\left(\mathrm{~d}, 1 \mathrm{H}, \mathrm{J}=6.3 \mathrm{~Hz}, \mathrm{C}_{7}-\mathrm{H}\right), 7.13$ (s, $1 \mathrm{H},-\mathrm{NH}, \mathrm{D}_{2} \mathrm{O}$ exchange), 7.16-7.20 (m, 3H, $\left.\mathrm{C}_{5}, \mathrm{C}_{6}, \mathrm{C}_{8}-\mathrm{H}\right), 7.44(\mathrm{~d}$, $\left.2 \mathrm{H}, \mathrm{J}=7.8 \mathrm{~Hz}, \mathrm{C}_{3}, \mathrm{C}_{5}, \mathrm{H}\right)$. Anal. Calcd. for $\mathrm{C}_{16} \mathrm{H}_{16} \mathrm{ClN}_{3} \mathrm{O}: \mathrm{C}, 63.68 ; \mathrm{H}$, 5.34; N, 13.92. Found: C, 63.51; H, 5.4; N, 13.89.

$\mathrm{N}$-(1,2,3,4-tetrahydroisoquinoline-2-yl)-Benzo[d]thiazole-2carboxamide (6v): Yield 5\%; mp 191.2-193.0 ${ }^{\circ} \mathrm{C}$; ${ }^{1} \mathrm{HNMR}\left(\mathrm{CDCl}_{3}\right)$ $\delta(\mathrm{ppm}): 3.12\left(\mathrm{t}, 2 \mathrm{H}, \mathrm{J}=6.0 \mathrm{~Hz}, \mathrm{C}_{4}-\mathrm{H}\right), 3.39\left(\mathrm{t}, 2 \mathrm{H}, \mathrm{J}=6.0 \mathrm{~Hz}, \mathrm{C}_{3}-\mathrm{H}\right)$, $4.28\left(\mathrm{~s}, 2 \mathrm{H}, \mathrm{C}_{1}-\mathrm{H}\right), 7.03\left(\mathrm{~d}, 1 \mathrm{H}, \mathrm{J}=1.8 \mathrm{~Hz}, \mathrm{C}_{7}-\mathrm{H}\right), 7.05(\mathrm{~s}, 1 \mathrm{H},-\mathrm{NH}$, $\mathrm{D}_{2} \mathrm{O}$ exchange), 7.14-7.21 (m, $\left.3 \mathrm{H}, \mathrm{C}_{5}, \mathrm{C}_{6}, \mathrm{C}_{8}-\mathrm{H}\right)$, 7.47-7.58 (m, $2 \mathrm{H}, \mathrm{C}_{7}$, $\left.\mathrm{C}_{8}-\mathrm{H}\right), 7.75\left(\mathrm{~d}, 1 \mathrm{H}, \mathrm{C}_{4^{4}}-\mathrm{H}\right), 7.97-8.03\left(\mathrm{~m}, 1 \mathrm{H}, \mathrm{C}_{9},-\mathrm{H}\right), 8.05-8.08(\mathrm{~m}, 1 \mathrm{H}$, $\mathrm{C}_{6}-\mathrm{H}$ ). Anal. Calcd. for $\mathrm{C}_{17} \mathrm{H}_{15} \mathrm{~N}_{3} \mathrm{OS} 0.05$ EtOAc: C, 65.42; H, 4.83; N, 13.36. Found: $\mathrm{C}, 65.07 ; \mathrm{H}, 4.82 ; \mathrm{N}, 13.39$.

6-(Trifluoromethyl)-N-(1,2,3,4-tetrahydroisoquinolin-2-yl)nicotinamide (6w): Yield 55\%; $\mathrm{mp}$ 172.6-174.3 ${ }^{\circ} \mathrm{C} ;{ }^{1} \mathrm{HNMR}\left(\mathrm{CDCl}_{3}\right) \delta$ (ppm): 3.10 (t, 2H, J=6.0 Hz, C $-\mathrm{H}), 3.43\left(\mathrm{t}, 2 \mathrm{H}, \mathrm{J}=6.0 \mathrm{~Hz}, \mathrm{C}_{3}-\mathrm{H}\right), 4.29$ $\left(\mathrm{s}, 2 \mathrm{H}, \mathrm{C}_{1}-\mathrm{H}\right), 7.03\left(\mathrm{~d}, 1 \mathrm{H}, \mathrm{J}=6 . .9 \mathrm{~Hz}, \mathrm{C}_{7}-\mathrm{H}\right), 7.12\left(\mathrm{~s}, 1 \mathrm{H},-\mathrm{NH}, \mathrm{D}_{2} \mathrm{O}\right.$ exchange), 7.14-7.18 (m, 3H, $\left.\mathrm{C}_{5}, \mathrm{C}_{6}, \mathrm{C}_{8}-\mathrm{H}\right), 7.75\left(\mathrm{~d}, 1 \mathrm{H}, \mathrm{C}_{4}-\mathrm{H}\right), 8.31(\mathrm{~d}$, $\left.1 \mathrm{H}, \mathrm{J}=8.4 \mathrm{~Hz}, \mathrm{C}_{5},-\mathrm{H}\right), 9.03\left(\mathrm{~s}, 1 \mathrm{H}, \mathrm{C}_{2},-\mathrm{H}\right)$. Anal. Calcd. for $\mathrm{C}_{16} \mathrm{H}_{14} \mathrm{~N}_{3} \mathrm{OF}_{3}$ $0.25 \mathrm{MeOH}$ : C, 58.63; H, 4.16; N, 12.695. Found: C, 58.36; H, 4.29; N, 12.76 .
2,6-Dichloro-5-fluoro- $\mathrm{N}-(1,2,3,4-t e t r a h y d r o i s o q u i n o l i n-2-y l)$ nicotinamide (6x): Yield 63\%; mp 169.5-171.0 ${ }^{\circ} \mathrm{C}$; ${ }^{1} \mathrm{HNMR}\left(\mathrm{CDCl}_{3}\right)$ $\delta$ (ppm): $3.09\left(\mathrm{t}, 2 \mathrm{H}, \mathrm{J}=6.3 \mathrm{~Hz}, \mathrm{C}_{4}-\mathrm{H}\right), 3.42\left(\mathrm{t}, 2 \mathrm{H}, \mathrm{J}=6.0 \mathrm{~Hz}, \mathrm{C}_{3}-\mathrm{H}\right)$, $4.26\left(\mathrm{~s}, 2 \mathrm{H}, \mathrm{C}_{1}-\mathrm{H}\right), 7.03\left(\mathrm{~d}, 1 \mathrm{H}, \mathrm{J}=6.9 \mathrm{~Hz}, \mathrm{C}_{7}-\mathrm{H}\right), 7.15(\mathrm{~s}, 1 \mathrm{H},-\mathrm{NH}$, $\mathrm{D}_{2} \mathrm{O}$ exchange), 7.16-7.20 (m, 3H, $\left.\mathrm{C}_{5}, \mathrm{C}_{6}, \mathrm{C}_{8}-\mathrm{H}\right), 7.92(\mathrm{~d}, 1 \mathrm{H}, \mathrm{J}=6.0 \mathrm{~Hz}$, $\left.\mathrm{C}_{4}-\mathrm{H}\right)$. Anal. Calcd. for $\mathrm{C}_{15} \mathrm{H}_{12} \mathrm{~N}_{3} \mathrm{OCl}_{2} \mathrm{~F}$ : C, 52.96; $\mathrm{H}, 3.56 ; \mathrm{N}, 12.35$. Found: C, 52.78; H, 3.60; N, 12.09 .

\section{Antiproliferative Activity Studies}

The antiproliferative activity of compound $6 a-x$ was evaluated at the Southern Research Institute (SRI, Birmingham, Alabama, USA) according to procedure [25]. The compounds were screened against human ER (+) MCF-7 (breast), ER (-) MDA-MB-231 (breast), and Ishikawa (endometrial) cancer cell lines in comparison to tamoxifen (TAM).

\section{Material}

Human MCF-7 and MDA-MB-231 breast cancer cell lines were purchased from the NCI. The human Ishikawa endometrial cancer cell line was purchased from Sigma Aldrich. All three cell lines were cultured in phenol red-free RPMI-1640 (Hyclone) $(500 \mathrm{~mL})$ supplemented with L-glutamine-dipeptide (Hyclone) $(5 \mathrm{~mL})$, and $10 \%$ fetal bovine serum (Atlanta Biologicals) $(50 \mathrm{~mL})$.

\section{Method}

The cell lines were cultured and treated with compounds under study including the standard TAM ranging from $0.01-100,000 \mathrm{nM}$ concentration in the presence of $10 \mathrm{nM}$ estradiol using the previous reported method [26]. The results expressed as $\mathrm{IC}_{50}$ (inhibitory concentration of $50 \%$ ) were the averages of three data points for each concentration and were calculated using GraphPad Prism 4.0.

\section{Molecular modeling studies}

Docking method: The crystal structures of ERa-4-OHT complex (PDB: 3ERT) and ER $\beta$-RAL complex (PDB:1QKN) whose coordinates were obtained from RCSB Protein Data Bank were used as a template to dock the active THIQs of present study The crystal structures was imported to Sybyl-X 1.3 [27] modeling suite and using Structure Preparation tool, Chain A was extracted, hydrogen atoms were added, MMFF94s force fields and MMFF94 charges were assigned to the atoms and energy minimized. The 3D structures of the THIQs and the co-crystallized ligands, 4-Hydroxytamoxifen (OHT), Raloxifene (RAL) were generated by Sybyl sketch and the conformer ensembles of all the compounds to be docked were generated using OMEGA v2.4.6, OpenEye Scientific Software $[28,29]$ prior to docking. OMEGA ensures that low strain energy conformations were retained in the ensemble. Since the complexes in the present study has bound ligands (OHT, RAL respectively), HYBRID v3.0.1 of OEDocking [30,31] was chosen as the appropriate docking method for our studies. Briefly, HYBRID docks molecules by an exhaustive search algorithm that systematically searches rotations and translations of each ligand conformation within the active site. In the process, unrealistic poses are filtered out, and those that survive are scored. The specified number of top scoring poses (in this study, 10), are subject to systematic solid body optimization. The best scoring pose is then used to rank the ligand against other ligands in the database. The protein and the conformers are held rigid during the docking process. Ligand flexibility is implicitly included by docking a conformer ensemble of each molecule and the bound cocrystal ligand information in the protein active site is used as a guide for docking 
the external ligand and scoring. The default parameters were set for HYBRID and OMEGA in this study. The scoring function used in this process to evaluate the poses in HYBRID is HYBRID_Chemgauss4 [32]. It uses Gaussian-smoothed potentials to measure the complementary nature of ligand poses within the active site.

Docking studies: The X-ray structure of Ligand Binding Domain of estrogen receptors has provided a way to better understand the ER binding site. The validation of the docking poses of the bound ligands (antagonists) in the crystal structures of ERa-4-OHT complex (3ERT) and ER $\beta$-RAL complex (1QKN) was done using OEdocking application, HYBRID [30,31]. The re-docking of the co-crystallized ligands has been undertaken to make sure that the bound conformations of the ligands (OHT, RAL) are reproduced by the selected docking method. All the best 10 poses retrieved through re-docking using HYBRID method were identical with the original poses of the cognate ligands in the crystal structures with root mean square deviation (rmsd) values between them being $<2 \mathrm{~A}^{\circ}$, a criterion often used for the correct bound structure prediction and validation. This led us to believe that the OEdocking method, HYBRID can be used reliably as a docking tool in our modeling studies.

\section{Results and Discussion}

\section{Chemistry}

Substituted tetrahydroisoquinoline derivatives $\mathbf{6 a}-\mathbf{x}$ were prepared according to the procedure depicted in Scheme 1. The amino salt, 2 -aminoisoquinolinium Iodide (3) used in the present study were prepared by the reaction of isoquinoline and hydroxylamine-Osulfonic acid and water, which was refluxed at $90^{\circ} \mathrm{C}$ for $2 \mathrm{~h}$ as previously reported procedure [33]. Reaction of (3) with corresponding substituted acylating agents like acylchlorides or sulfonyl chlorides, followed by treatment with a base afforded $\mathrm{N}$-ylides $\mathbf{5}$ as stable crystalline solids. Sodium borohydride reduction of $\mathbf{5 a}-\mathbf{x}$ in absolute ethanol furnished the title compounds $\mathbf{6 a - x}$ in fair to good yields. It should be noted that some of the compounds synthesis have been reported [34-36]. However, none of these compounds has been examined for their antiproliferative activities on breast cancer cells.

\section{Antiproliferative activity}

In vitro antiproliferative activity of compounds $\mathbf{6 a - x}$ were evaluated against on human MDA-MB-231 (ER negative breast carcinoma cell line), MCF-7 (ER positive breast cancer cell line) and Ishikawa (endometrial) cancer cell lines at concentration ranging from 0.01$100,000 \mathrm{nM}$ in the presence of $10 \mathrm{nM}$ estradiol (E2) using CellTiterGlo assay (E2 was used for competitive growth inhibitory studies). As shown in Table 1, compounds 6a-f, 6i-k, $60\left(\mathrm{IC}_{50}=0.4-3.5 \mu \mathrm{g} / \mathrm{mL}\right)$ demonstrated significant antiproliferative activity against human $\mathrm{Er}$ (+) MCF-7 breast cancer cell line in comparison to TAM $\left(\mathrm{IC}_{50}=5.14 \mu \mathrm{g} /\right.$ $\mathrm{mL}$ ). (Note: $\mathrm{IC}_{50}$ is the concentration of test drug where a $50 \%$ reduction is observed in cell growth compared to the untreated control after a 72 $\mathrm{h}$ period of exposure to test drug). Compounds bearing phenyl (6a) and ethyl (6d) groups, the most active of the series in this cell line, exhibit higher antiproliferative activity against MCF-7 cell line based on $\mathrm{IC}_{50}$ value (Figure 1).

The ER (-) MDA-MB-231 breast cancer cell line constitutes an original model for identifying the ER-independent mechanisms of TAM antiproliferative effects $[37,38]$. Thus, in the present study the antiproliferative activity of compounds $\mathbf{6 a - x}$ against human ER (-) MDA-MB-231 breast cancer cell lines were also investigated to know their mechanism of action. The results showed that compounds 6 ae, 6j-k and $6 \mathbf{0}\left(\mathrm{IC}_{50}=0.3-4.85 \mu \mathrm{g} / \mathrm{mL}\right)$ were more potent than TAM $\left(\mathrm{IC}_{50}=5.64 \mu \mathrm{g} / \mathrm{mL}\right.$ ) shown in Table 1 . Furthermore compound $\mathbf{6 d}$ $\left(\mathrm{IC}_{50}=0.37 \mu \mathrm{g} / \mathrm{mL}\right)$ possessing ethyl group on phenyl ring, the most active of the series in this cell line. This results suggests that compound $6 \mathrm{~d}$ may also inhibit cell proliferation via ER-independent mechanism in comparison to TAM (Figure 2).

In the Present investigation, evaluation of the antiproliferative activity of these compounds against human Ishikawa endometrial cell line were revealed that compounds $\mathbf{6 a}, \mathbf{6 b}, \mathbf{6} \mathbf{d}$ and $\mathbf{6 j}\left(\mathrm{IC}_{50}=0.23\right.$, $0.21,0.01$ and $0.02 \mu \mathrm{g} / \mathrm{mL}$ respectively) were more potent than TAM $\left(\mathrm{IC}_{50}=4.55 \mu \mathrm{g} / \mathrm{mL}\right.$, Table 1). These results indicate that compounds $\mathbf{6 d}$ may lower the risk of developing uterine cancer based upon the $\mathrm{IC}_{50}$ value in comparison with TAM [26] (Figure 3).

\section{Molecular modeling studies}

The top scoring conformations of the THIQs $(\mathbf{6 a - 6 k}, 6 \mathbf{6}, \mathbf{6 r}, \mathbf{6 t}$ and $\mathbf{6 v}-\mathbf{6 x}$ ) collected by docking the conformer ensemble (generated by OMEGA $[28,29]$ ) on ER $\alpha$ and ER $\beta$ receptors (Figures 4 and 5) clearly show the preference for ERa as the ligands fit better in the bigger ligand binding pocket of ERa. These studies also give us an idea of the probable bioactive conformations and binding mode of the newly synthesized THIQs which would assist in further optimization studies. Around

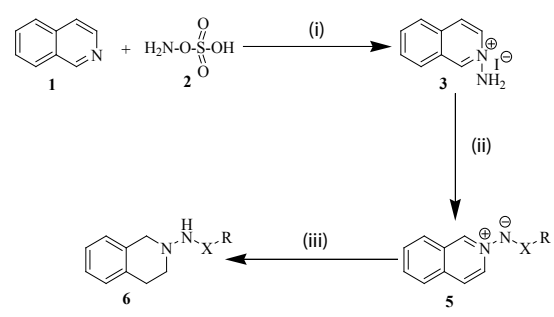

Reaction Conditions: (i) $\mathrm{H}_{2} \mathrm{O}, 90^{\circ} \mathrm{C}, 2 \mathrm{~h}, \mathrm{~K}_{2} \mathrm{CO}_{3} \mathrm{HI}$; (ii) R-X-Cl (4)Dry THF, $\mathrm{Et}_{3} \mathrm{~N}$, refulx, $12 \mathrm{~h}$ (iii) $\mathrm{NaBH}_{4}$, abs EtOH, $0^{\circ} \mathrm{C}, 7 \mathrm{~h} ; \mathrm{R}=\mathrm{C}_{6} \mathrm{H}_{5}, 4-\mathrm{CH}_{3}-\mathrm{C}_{6} \mathrm{H}_{4}, 4-\mathrm{OCH}_{3}-\mathrm{C}_{6} \mathrm{H}_{4}, 4-\mathrm{Cl}_{-} \mathrm{C}_{6} \mathrm{H}_{4} ; 4-\mathrm{Br}_{-} \mathrm{C}_{6} \mathrm{H}_{4}, 4-\mathrm{F}-\mathrm{C}_{6} \mathrm{H}_{4}$ $4-\mathrm{C}_{2} \mathrm{H}_{5}-\mathrm{C}_{6} \mathrm{H}_{4}, 4-\mathrm{C}_{3} \mathrm{H}_{7}-\mathrm{C}_{6} \mathrm{H}_{4}, 4-\mathrm{C}_{4} \mathrm{H}_{9}-\mathrm{C}_{6} \mathrm{H}_{4}, 4-\mathrm{C}\left(\mathrm{CH}_{3}\right)_{3}-\mathrm{C}_{6} \mathrm{H}_{4}, 3,4,5-$ tri- $_{6} \mathrm{OCH}_{3}-\mathrm{C}_{6} \mathrm{H}_{2}, 4-\mathrm{CF}_{3}-\mathrm{C}_{6} \mathrm{H}_{4}, 4-\mathrm{NO}_{2}-$ $\mathrm{C}_{6} \mathrm{H}_{4}, 2$-furfuryl, 6-Cl- $\mathrm{C}_{5} \mathrm{H}_{3} \mathrm{~N}, \mathrm{X}=\mathrm{CO}, \mathrm{SO}_{2}$.

Scheme 1

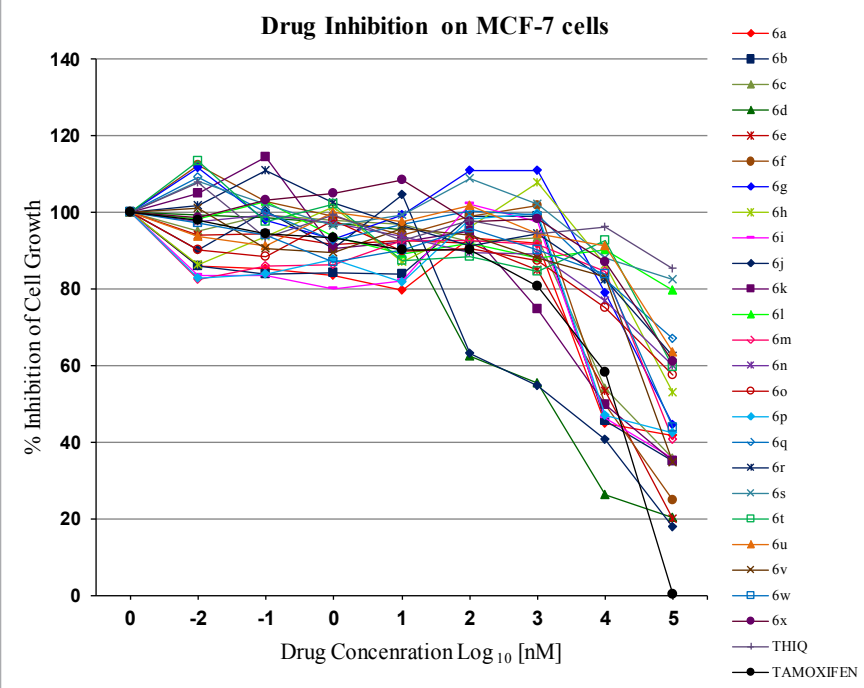

Figure 1: In vitro antiproliferative activity of compounds $6 a-x$ against ER (+) MCF-7 cell line. 


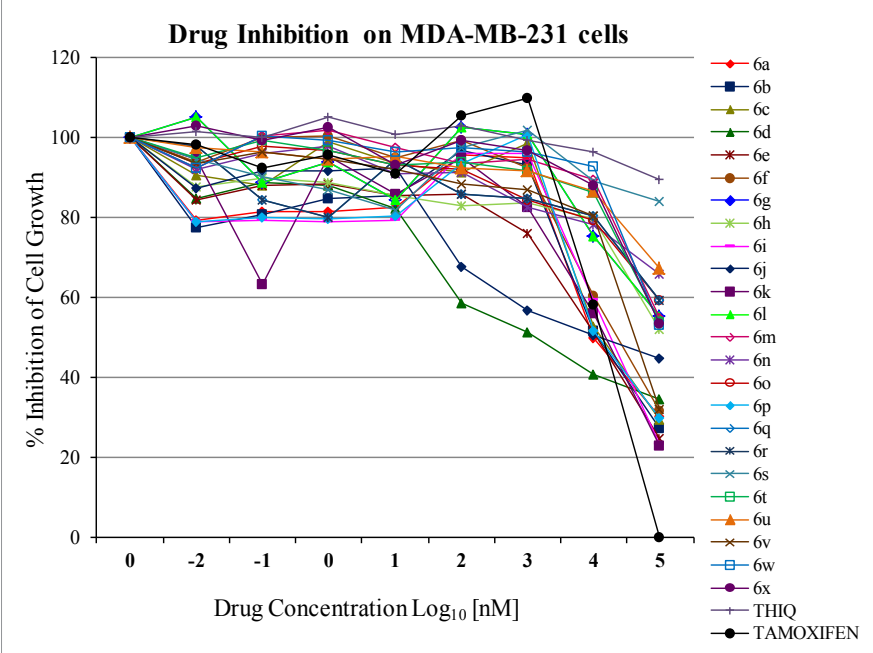

Figure 2: In vitro antiproliferative activity of compounds 6a-x against ER (-) MDA-MB-231 cell line.

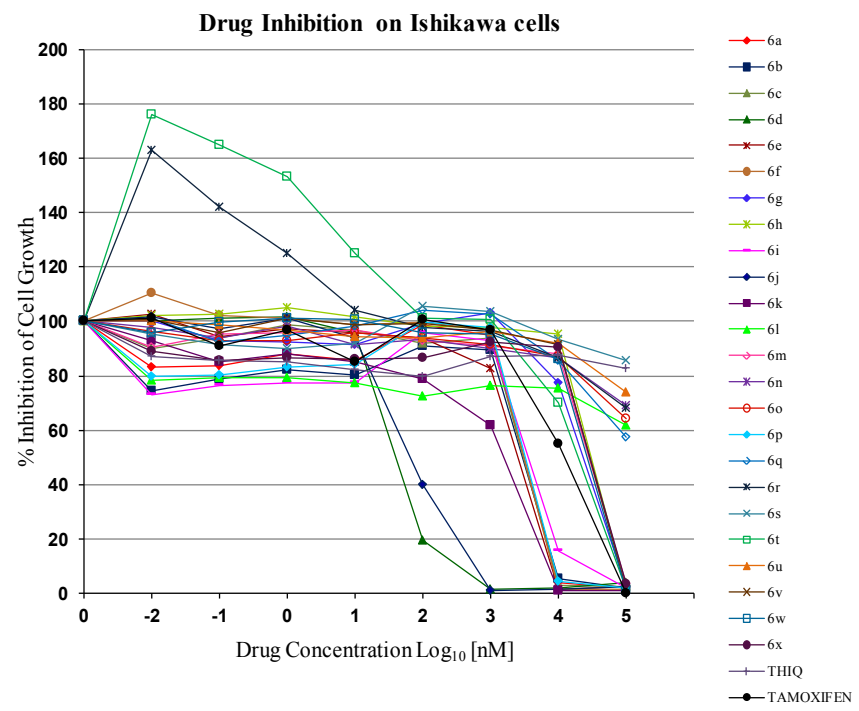

Figure 3: In vitro antiproliferative activity of compounds 6a-x against Ishikawa cell line.

ten tetrahydroisqoquinolines (THIQs) synthesized were more potent than Tamoxifen on all the cell lines understudy. The best being the THIQ, 6d as shown in Table 1, the high potency of these compounds on Ishikawa cell lines as compared with Tamoxifen, indicate that these compounds may lower the risk of developing uterine cancer. Tetrahydroisoquinolines were reported as subtype selective estrogen agonists/antagonists as they are structurally similar to Lasofoxifene. The selectivity towards ERa and ER is crucial, as they have their own tissue distribution patterns and transcriptional properties. ERa is mainly involved in reproduction events in uterus and mammary glands and $\operatorname{ER} \beta$ is more generally expressed and not dominant in uterus and breast tissues.

\section{Discussion}

The antiproliferative activity of twenty four substituted tetrahydroisoquinoline analogs $(\mathbf{6 a}-\mathbf{x})$ were evaluated in two human breast cancer cell lines (MCF-7, MDA-MB-231) and human endometrial cancer cell lines (Ishikawa) in order to understand their underlying mechanism of action of their antiproliferative activity. This study also helps us to evaluate the effect of various substitutions on the phenyl ring of the THIQs (Scheme 1) towards antiproliferative activity. In this regard, high-quality biological testing results were collected for the newly synthesized compounds along with Tamoxifen (Table $\mathbf{1}$ ). The most active compounds are $\mathbf{6 a}, \mathbf{6 b}, \mathbf{6 c}, \mathbf{6 d}, \mathbf{6 i}, \mathbf{6 j}, \mathbf{6 k}$ and $\mathbf{6 p}$. These results strongly indicate that the antiproliferative activity of most of our compounds on Ishikawa cell lines were far better than the standard Tamoxifen, indicating that the compounds in the present study have low associated risk in developing endometrial cancer and its treatment. The active compounds mentioned above showed nearly similar activity on MCF-7 and MDA-MB-231 cell lines, except for $\mathbf{6 j}$ where the compound is more active on MCF-7 cell lines than MDA-MB-231. These results indicate that except for compound $\mathbf{6 j}$, all other active compounds in the present study may also inhibit cell proliferation

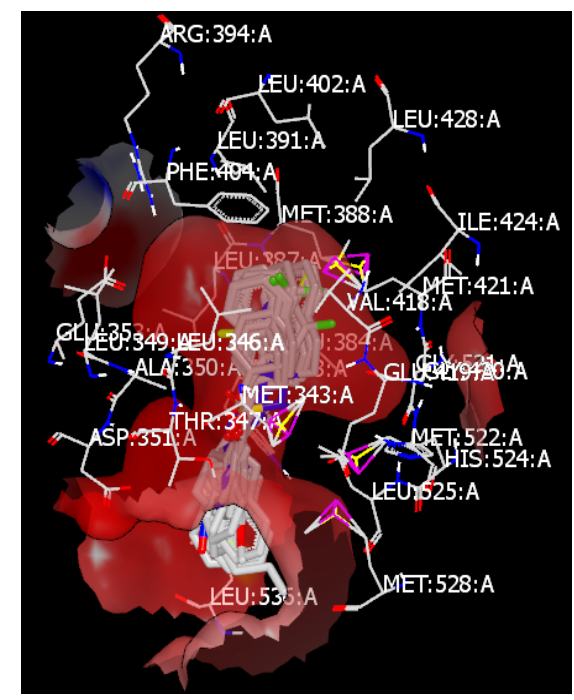

Figure 4: Top scoring binding poses of the active THIQs $(6 a-6 \mathrm{k}, 60,6 \mathrm{r}$, 6t and $6 \mathrm{v}-6 \mathrm{x})$ at the active site of ERa-4-OHT complex (3ERT).

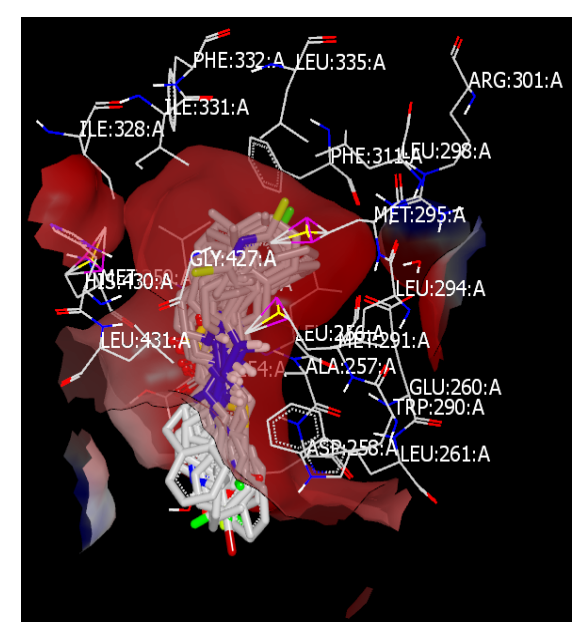

Figure 5: Top scoring binding poses of the active THIQs (6a-6k, 6o, 6r, 6t and $6 v-6 x)$ at the active site of ER $\beta-R A L$ complex (1QKN). 


\begin{tabular}{|c|c|c|c|c|c|}
\hline \multirow{2}{*}{ CODE } & \multirow{2}{*}{$\mathbf{R}$} & \multirow{2}{*}{$\mathbf{x}$} & \multicolumn{3}{|c|}{$\mathrm{IC}_{50} \mu \mathrm{g} / \mathrm{mL}^{1,2}$} \\
\hline & & & MCF-7 & ISHIKAWA & MDA-MB-231 \\
\hline $6 a$ & $\mathrm{C}_{6} \mathrm{H}_{5}$ & $\mathrm{CO}$ & 0.63 & 0.23 & 0.74 \\
\hline $6 \mathrm{~b}$ & $4-\mathrm{CH}_{3}-\mathrm{C}_{6} \mathrm{H}_{4}$ & $\mathrm{CO}$ & 0.70 & 0.44 & 1.02 \\
\hline $6 c$ & $4-\mathrm{OCH}_{3}-\mathrm{C}_{6} \mathrm{H}_{4}$ & $\mathrm{CO}$ & 0.93 & 0.21 & 0.78 \\
\hline $6 d$ & $4-\mathrm{C}_{2} \mathrm{H}_{5}-\mathrm{C}_{6} \mathrm{H}_{4}$ & $\mathrm{CO}$ & 0.43 & 0.01 & 0.37 \\
\hline $6 e$ & $4-\mathrm{C}_{3} \mathrm{H}_{7}-\mathrm{C}_{6} \mathrm{H}_{4}$ & $\mathrm{CO}$ & 3.71 & 0.75 & 3.26 \\
\hline $6 f$ & $4-\mathrm{C}_{4} \mathrm{H}_{9}-\mathrm{C}_{6} \mathrm{H}_{4}$ & $\mathrm{CO}$ & 3.05 & 0.96 & 7.03 \\
\hline $6 \mathrm{~g}$ & 4-tert- $\mathrm{C}_{4} \mathrm{H}_{9}-\mathrm{C}_{6} \mathrm{H}_{4}$ & $\mathrm{CO}$ & 21.34 & 7.12 & $>30.84$ \\
\hline $6 \mathrm{~h}$ & $4-\mathrm{F}-\mathrm{C}_{6} \mathrm{H}_{4}$ & $\mathrm{CO}$ & $>27.03$ & 8.3 & $>27.03$ \\
\hline $6 i$ & $4-\mathrm{Cl}-\mathrm{C}_{6} \mathrm{H}_{4}$ & $\mathrm{CO}$ & 0.71 & 0.61 & 0.80 \\
\hline $6 j$ & $4-\mathrm{Br}-\mathrm{C}_{6} \mathrm{H}_{4}$ & $\mathrm{CO}$ & 0.7 & 0.02 & 4.34 \\
\hline $6 k$ & $4-\mathrm{CF}_{3}-\mathrm{C}_{6} \mathrm{H}_{4}$ & $\mathrm{CO}$ & 3.15 & 0.5 & 4.85 \\
\hline 61 & 3,4,5-tri- $\mathrm{OCH}_{3}-\mathrm{C}_{6} \mathrm{H}_{2}$ & $\mathrm{CO}$ & $>34.24$ & $>34.24$ & $>34.24$ \\
\hline $6 \mathrm{~m}$ & $4-\mathrm{NO}_{2}-\mathrm{C}_{6} \mathrm{H}_{4}$ & $\mathrm{CO}$ & 18.23 & 8.39 & $>29.73$ \\
\hline $6 n$ & $\mathrm{C}_{6} \mathrm{H}_{5}$ & $\mathrm{SO}_{2}$ & $>28.84$ & $>28.84$ & $>28.84$ \\
\hline 60 & $4-\mathrm{CH}_{3}-\mathrm{C}_{6} \mathrm{H}_{4}$ & $\mathrm{SO}_{2}$ & $>30.24$ & $>30.24$ & $>30.24$ \\
\hline $6 p$ & $4-\mathrm{OCH}_{3}-\mathrm{C}_{6} \mathrm{H}_{4}$ & $\mathrm{SO}_{2}$ & 1.27 & 0.45 & 0.76 \\
\hline $6 q$ & $4-\mathrm{C}_{2} \mathrm{H}_{5}-\mathrm{C}_{6} \mathrm{H}_{4}$ & $\mathrm{SO}_{2}$ & $>31.64$ & $>31.64$ & $>31.64$ \\
\hline $6 r$ & 4- $\mathrm{C}_{4} \mathrm{H}_{9}-\mathrm{C}_{6} \mathrm{H}_{4}$ & $\mathrm{SO}_{2}$ & $>34.45$ & $>34.45$ & $>34.45$ \\
\hline $6 s$ & $2-\mathrm{C}_{4} \mathrm{H}_{3} \mathrm{O}$ & $\mathrm{CO}$ & $>24.23$ & $>24.23$ & $>24.23$ \\
\hline $6 \mathrm{t}$ & $6-\mathrm{Cl}-\mathrm{C}_{5} \mathrm{H}_{3} \mathrm{~N}$ & $\mathrm{CO}$ & $>28.77$ & 5.62 & $>28.77$ \\
\hline $6 u$ & 2-Cl-6- $\mathrm{CH}_{3}-\mathrm{C}_{5} \mathrm{H}_{2} \mathrm{~N}$ & $\mathrm{CO}$ & $>30.18$ & $>30.18$ & $>30.18$ \\
\hline $6 v$ & $\mathrm{C}_{7} \mathrm{H}_{5} \mathrm{NS}$ & $\mathrm{CO}$ & 15.0 & 8.98 & 13.11 \\
\hline $6 w$ & $6-\mathrm{CF}_{3}-\mathrm{C}_{5} \mathrm{H}_{3} \mathrm{~N}$ & $\mathrm{CO}$ & 22.51 & 8.57 & $>32.13$ \\
\hline $6 x$ & 2,6-di-Cl-5-F- $\mathrm{C}_{5} \mathrm{HN}$ & $\mathrm{CO}$ & $>34.02$ & 9.95 & $>34.02$ \\
\hline THIQ & & - & $>13.32$ & $>13.32$ & $>13.32$ \\
\hline & TAMOXIFEN & & 5.14 & 4.55 & 5.64 \\
\hline
\end{tabular}

${ }^{1}$ The data represent the average of triplicate determinations at various concentrations

${ }^{2} \mathrm{The} \mathrm{IC}_{50}$ values were determined from the graphs (GraphPad Prism) using mean values of data points at various concentrations

Table 1: $\mathrm{IC}_{50}$ values $(\mu \mathrm{g} / \mathrm{mL}$ ) for compounds 6a-x tested against MCF-7, MDA-MB231 and Ishikawa Cancer Cells.

via ER-independent mechanism in comparison to TAM. As far as the structure activity relationship is concerned, ethyl group with the right steric bulk, halogens, $\mathrm{OCH}_{3}$ and $\mathrm{CH}_{3}$ groups at the -para position $\left(4^{\text {th }}\right.$ position) of the aromatic ring is the cause of activity. Replacing the phenyl right with other heterocycles and additional ring substitutions on the phenyl ring lead to loss of activity. Similarly, hetero atoms incorporated inside the aromatic ring as in led to loss in activity.

\section{Conclusion}

The newly synthesized substituted tetrahydroisoquinolies (THIQs) were characterized thoroughly by ${ }^{1} \mathrm{H}$ NMR and elemental analysis to make sure the purity of these compounds for in vitro testing. Their antiproliferative activities against human $\mathrm{ER}(+)$ MC-7 (breast), ER (-) MDA-MB-231 (breast) and Ishikawa (endometrial) cell lines were determined and compared to that of standard antiestrogen drug TAM. In vitro results indicated that some of the compounds in the study $\mathbf{6 a}$, $\mathbf{6 b}, \mathbf{6 c}, \mathbf{6 d}, \mathbf{6 i}, \mathbf{6 j} \mathbf{6 k}$, and $\mathbf{6 p}$ showed better activity than TAM on the above cell lines. Compound $\mathbf{6 j}$ showed better selectivity on MCF-7 cell lines over MDA-MB-231 cells lines indicating a possible ER-dependent antiproliferative action. Compound $\mathbf{6 d}$ with an ethyl substitution on the 4 th position of the THIQ phenyl ring was the best compound in this series for future studies with $\mathrm{IC}_{50}$ values on MCF-7 and MDA-MB-231 being 0.43 and $0.37 \mu \mathrm{g} / \mathrm{mL}$ respectively. Pose prediction of the active compounds were undertaken by docking these compounds on the published crystal structures of ER- $\alpha$ and ER- $\beta$ receptors. These in silico preliminary studies indicate a preference for these compounds to the relatively larger ER- $\alpha$ binding site. The future studies in this direction involve exploring the activity profile of this class of compounds by substitutions on the THIQ ring keeping the ethyl group intact on the 4-th position of the phenyl ring, ER binding studies, uterotropic and antiuterotropic activities on immature rats in order to further understand how these compounds interact with ER.

\section{Acknowledgements}

We are grateful to the National Center for Research Resources and the National Institute of Minority Health and Health Disparities of the National Institutes of Health through Grant Number 8 G12MD007582-28. We are also grateful to Dr. Michael J. Roberts, Cell Biology and Immunology, Southern Research Institute, Birmingham, AL, for testing cytotoxicity on there cell lines and OpenEye Scientific software, Inc., Santa Fe, NM for free Academic License.

\section{References}

1. Landis MD, Lehmann BD, Pietenpol JA, Chang JC (2013) Patient-derived breast tumor xenografts facilitating personalized cancer therapy. Breast Cancer Res 15: 201.

2. Osborne CK, Schiff R, Arpino G, Lee AS, Hilsenbeck VG (2005) Endocrine responsiveness: understanding how progesterone receptor can be used to select endocrine therapy. Breast 14: 458-465.

3. Johnston S (2004) Fulvestrant and the sequential endocrine cascade for advanced breast cancer. Br J Cancer 90 Suppl 1: S15-18.

4. (1998) Tamoxifen for early breast cancer: an overview of the randomised trials Early Breast Cancer Trialists' Collaborative Group. Lancet 351: 1451-1467.

5. Jordan VC (1992) The role of tamoxifen in the treatment and prevention of breast cancer. Curr Probl Cancer 16: 129-176. 
Citation: Gangapuram M, Eyunni S, Redda KK (2014) Synthesis and Pharmacological Evolution of Tetrahydroisoquinolines as Anti Breast Cancer Agents. J Cancer Sci Ther 6: 161-169. doi:10.4172/1948-5956.1000266

6. Lerner LJ, Jordan VC (1990) Development of antiestrogens and their use in breast cancer: eighth Cain memorial award lecture. Cancer Res 50: 4177-4189.

7. Ettinger B (1998) Overview of estrogen replacement therapy: a historical perspective. Proc Soc Exp Biol Med 217: 2-5.

8. Brett KM, Madans JH (1997) Use of postmenopausal hormone replacement therapy: estimates from a nationally representative cohort study. Am J Epidemiol 145: 536-545.

9. Hubber JC (2001) New directions in HRT. ORGYN: 3-5.

10. el Amouri H, Vessières A, Vichard D, Top S, Gruselle M, et al. (1992) Syntheses and affinities of novel organometallic-labeled estradiol derivatives: a structureaffinity relationship. J Med Chem 35: 3130-3135.

11. Ahmed N, Dubuc C, Rousseau J, Bénard F, van Lier JE (2007) Synthesis characterization, and estrogen receptor binding affinity of flavone-, indole-, and furan-estradiol conjugates. Bioorg Med Chem Lett 17: 3212-3216.

12. Osborne CK, Coronado-Heinsohn EB, Hilsenbeck SG, McCue BL, Wakeling $A E$, et al. (1995) Comparison of the effects of a pure steroidal antiestrogen with those of tamoxifen in a model of human breast cancer. J Natl Cancer Inst 87: 746-750.

13. Bowler J, Lilley TJ, Pittam JD, Wakeling AE (1989) Novel steroidal pure antiestrogens. Steroids 54: 71-99.

14. Jordan VC (1992) The strategic use of antiestrogens to control the development and growth of breast cancer. Cancer 70: 977-982.

15. Kuiper GG, Carlsson B, Grandien K, Enmark E, Häggblad J, et al. (1997) Comparison of the ligand binding specificity and transcript tissue distribution of estrogen receptors alpha and beta. Endocrinology 138: 863-870.

16. Seeger H, Huober J, Wallwiener D, Mueck AO (2004) Inhibition of human breast cancer cell proliferation with estradiol metabolites is as effective as with tamoxifen. Horm Metab Res 36: 277-280.

17. Fisher B, Costantino JP, Wickerham DL, Redmond CK, Kavanah M, et al. (1998) Tamoxifen for prevention of breast cancer: report of the National Surgical Adjuvant Breast and Bowel Project P-1 Study. J Natl Cancer Inst 90: 1371-1388.

18. Phillips DH, Venitt S (1993) Safety of prophylactic tamoxifen. Lancet 341: 14851486.

19. Jordan VC (2003) Tamoxifen: a most unlikely pioneering medicine. Nat Rev Drug Discov 2: 205-213.

20. Chesworth R, Zawistoski MP, Lefker BA, Renaud J, Bischoff SF, et al. (2003) Estrogen receptor modulators: identification and structure-activity relationships of potent ERalpha-selective tetrahydroisoquinoline ligands. J Med Chem 46: 2945-2957.

21. Lin HR, Safo MK, Abraham DJ (2007) Identification of a series of tetrahydroisoquinoline derivatives as potential therapeutic agents for breast cancer. Bioorg Med Chem Lett 17: 2581-2589.

22. Renaud J, Bischoff SF, Buhl T, Floersheim P, Fournier B, et al. (2003) Estrogen receptor modulators: identification and structure-activity relationships of poten ERalpha-selective tetrahydroisoquinoline ligands. J Med Chem 46: 2945-2957.

23. Renaud J, Bischoff SF, Buhl T, Floersheim P, Fournier B, et al. (2005) Selective estrogen receptor modulators with conformationally restricted side chains. Synthesis and structure-activity relationship of ERalpha-selective tetrahydroisoquinoline ligands. J Med Chem 48: 364-379.

24. Leese MP, Jourdan F, Dohle W, Kimberley MR, Thomas MP, et al. (2012) Steroidomimetic Tetrahydroisoquinolines for the Design of New Microtubule Disruptors. ACS Med Chem Lett 3: 5-9.

25. Crouch SP, Kozlowski R, Slater KJ, Fletcher J (1993) The use of ATP bioluminescence as a measure of cell proliferation and cytotoxicity. J Immuno Methods 160: 81-88

26. Suresh VK, Madhavi G, Redda KK (2014) In-vitro Antiproliferative Activity of New Tetrahydroisoquinolines (THIQs) on Ishikawa Cells and their 3D Pharmacophore Models. Letters in Drug Design \& Discovery 11: 428-436.

27. SYBYL-X 1.3, Tripos International.1699 S. Hanley Rd., St. Louis, MO, 63144 USA.

28. Hawkins PCD, Skillman AG, Warren GL, Ellingson BA, Stahl MT, OMEGA 2.4.6: OpenEye Scientific Software, Santa Fe, NM.

29. Hawkins PC, Skillman AG, Warren GL, Ellingson BA, Stahl MT (2010) Conformer generation with OMEGA: algorithm and validation using high quality structures from the Protein Databank and Cambridge Structural Database. J Chem Inf Model 50: 572-584.

30. McGann M, OEDOCKING 3.0.1. : OpenEye Scientific Software, Santa Fe, NM.

31. McGann M (2012) FRED and HYBRID docking performance on standardized datasets. J Comput Aided Mol Des 26: 897-906.

32. McGann MR, Almond HR, Nicholls A, Grant JA, Brown FK (2003) Gaussian docking functions. Biopolymers 68: 76-90.

33. Wallace RG (1980) Hydroxylamine-O-sulfonic acid- a versatile synthetic reagent. Aldrichchimica Acta 13: 3.

34. Powell BF, Overberger CG, Anselme JP (1983) Hydrosulfite Reduction of $\mathrm{N}$-Nitroso-1,2,3,4-tetrahdydroisoquinolines and Oxidation of N-Amino-1,2,3,4tetrahydroisoquinolines J Heterocyclic Chem 20: 121-128.

35. Höft E, Rieche A (1961) Darstellung von N-Amino-1.2.3.4-tetrahydroisochinolin und seine Oxydation. Angew Chem 73: 807-808.

36. Redda, KK, Gangapuram M, Ardley TW (2010) Synthesis of substituted 1,2,3,4-tetrahydroisoquinolines as anticancer agents, Proceedings of the 101st Annual Meeting of the American Association for Cancer Research, Washington DC. USA, 735

37. Colletta AA, Benson JR, Baum M (1994) Alternative mechanisms of action of anti-oestrogens. Breast Cancer Res Treat 31: 5-9.

38. Butta A, MacLennan K, Flanders KC, Sacks NP, Smith I, et al. (1992) Induction of transforming growth factor beta 1 in human breast cancer in vivo following tamoxifen treatment. Cancer Res 52: 4261-4264. 